\title{
Numerical Equilibrium Analysis for Structured Consumer Resource Models
}

\author{
A.M. de Roos ${ }^{\text {a }}$, O. Diekmann ${ }^{\text {b }}$, P. Getto ${ }^{\text {c,* }}$, M.A. Kirkilionis ${ }^{d}$ \\ ${ }^{a}$ Institute for Biodiversity and Ecosystem Dynamics (IBED), University of Amsterdam, \\ P.O. Box 94084, 1090GB Amsterdam, The Netherlands \\ ${ }^{b}$ Department of Mathematics, University of Utrecht, Budapestlaan 6, P.O. Box 80010, \\ 3508 TA Utrecht, The Netherlands \\ ${ }^{c}$ BCAM-Basque Center for Applied Mathematics, Bizkaia Technology Park, 48160 Derio, \\ Bizkaia, Spain \\ ${ }^{d}$ Department of Mathematics, University of Warwick, CV4 7AL Coventry, UK
}

Received: 3 March 2009 / Accepted: 16 July 2009 / Published online: 31 July 2009

(C) The Author(s) 2009. This article is published with open access at Springerlink.com

\begin{abstract}
In this paper, we present methods for a numerical equilibrium and stability analysis for models of a size structured population competing for an unstructured resource. We concentrate on cases where two model parameters are free, and thus existence boundaries for equilibria and stability boundaries can be defined in the (two-parameter) plane. We numerically trace these implicitly defined curves using alternatingly tangent prediction and Newton correction. Evaluation of the maps defining the curves involves integration over individual size and individual survival probability (and their derivatives) as functions of individual age. Such ingredients are often defined as solutions of ODE, i.e., in general only implicitly. In our case, the right-hand sides of these ODE feature discontinuities that are caused by an abrupt change of behavior at the size where juveniles are assumed to turn adult. So, we combine the numerical solution of these ODE with curve tracing methods. We have implemented the algorithms for "Daphnia consuming algae" models in C-code. The results obtained by way of this implementation are shown in the form of graphs.
\end{abstract}

Keywords Numerical equilibrium analysis $\cdot$ Structured populations $\cdot$ Stability boundaries · Hopf bifurcation · Consumer resource models - Delay equations - Renewal equations · Delay differential equations · Daphnia models

\section{Introduction}

In this paper, we present methods for a numerical equilibrium and stability analysis of a class of models of the interaction between a structured consumer population and an unstructured resource population.

\footnotetext{
*Corresponding author.

E-mail address: getto@bcamath.org (P. Getto).
} 
In unstructured consumer resource models and in predator prey models, one typically finds a Hopf-bifurcation marking the destabilization of an interior equilibrium and the emergence of a stable limit cycle (Rosenzweig, 1971). In de Roos et al. (1990), one finds a model of a size structured population of Daphnia magna consuming algae, which is parameterized on the basis of experimental data. Based on extensive numerical calculations, it is concluded in de Roos et al. (1990) that incorporating size structure of the consumer induces additional features, namely the possibility of the coexistence of a stable and an unstable limit cycle and even the coexistence of two stable limit cycles.

In Diekmann et al. (2007), a theoretical framework is described that establishes existence and uniqueness, the principle of linearized stability and the Hopf-bifurcation theorem for a class of abstract integral equations. For given resource, the dynamics of a structured population can be described by a renewal equation; see Metz and Diekmann (1986). This equation can also be classified as a Volterra integral equation or a functional equation of delay type (with continuously distributed delay); see Diekmann et al. (1995). Incorporating competition then amounts to adding an equation for the resource dynamics (Diekmann et al., 2007). This equation has on the right-hand side a similar structure as the renewal equation, but the left-hand side consists of the time derivative of the unknown resource concentration, whereas in the case of the renewal equation, it consists of the unknown function itself. It hence should be called an integro-differential equation or a delay differential equation (again with continuously distributed delay). Hence, in summary, we shall speak of a system of delay equations, a renewal equation coupled to a delay differential equation. Such systems are equivalent to special cases of the mentioned abstract integral equations; see again (Diekmann et al., 2007). In de Roos et al. (2009), the mathematical setting of Diekmann et al. (2007) is used to establish an analytical stability and bifurcation theory for structured consumer resource models with special attention for the Daphnia model in de Roos et al. (1990). Our aim here is to complement the analytical theory in de Roos et al. (2009) with tools for the numerical equilibrium and stability analysis of such models. Even though we use Daphnia models to test our algorithms, our approach works for more general structured consumer resource models. Moreover, we use formulations that should be easy to generalize to other structured population models.

As key results of the analysis of qualitative behavior, in de Roos et al. (1990) curves in two-parameter space marking stability boundaries where Hopf-bifurcations occur were presented. Integrals, which arise naturally when modeling structured populations, could in the case of the Daphnia model be evaluated analytically, and thus the curves could be approximated by coding established predictor- corrector methods (Allgower and Georg, 1990) without it being necessary to refer to numerical integration. We present a method here to compute stability boundaries for models specified in terms of general vital rates. Integrals involving these general vital rates can be evaluated by integration of a system of coupled ODE, which is generally not possible by hand. The idea, which can be found already in Kirkilionis et al. (2001), is to combine the curve tracing methods with numerical integration of the ODE. We use this idea here to establish methods to compute existence and stability boundaries for structured consumer resource models formulated as systems of delay equations.

The Daphnia models in de Roos et al. (1990) and (2009) exhibit a discontinuity of the consumers vital rates at the size at which juveniles turn adult, caused by an abrupt change of behavior, notably an abrupt onset of reproduction, upon reaching this size. We therefore allow also here for such discontinuities. 
Much of the length of this paper is caused by the complexity of the characteristic equation. This complexity is due to the fact that the following issues are involved in its computation:

- differentiation of solutions of ODE with respect to infinite dimensional parameters,

- discontinuities at the size where adulthood is reached,

- the rewriting of ingredients of the characteristic equation as solutions of (real) ODE.

In de Roos et al. (2009), an expression for the ingredients of the characteristic equation for our class of structured consumer resource models is given. Our present derivation of the characteristic equation is much inspired by this paper, but we found that for the numerical computation of the elements a different representation of the characteristic equation is more convenient.

In Section 2, we introduce the analytic setting for a class of models describing the interaction of a structured consumer population with an unstructured resource population. We formulate the population equations and discuss the existence of a unique interior equilibrium. Then we concentrate on the case where two model parameters are free and define existence boundaries for the equilibrium in the (two-parameter) plane. In this plane, we then define a curve marking Hopf-bifurcation points. The main ingredient here is the characteristic equation obtained via linearization of the population equations.

In Section 3, we present methods to numerically approximate the curves defined in Section 2. We start with an algorithm for the numerical integration involved in the approximation of equilibria. In a further algorithm, we show how to achieve this approximation by a combination of the numerical integration method and a Newton-method. In the case of two free parameters, we show how the curves can be continued by combining first tangent prediction and then correction with a Newton method with numerical integration. Here, the map defining stability boundaries is much more involved than the one defining existence boundaries, due to the already mentioned complexity of the characteristic equation.

In Section 4, we present the results obtained via the implementation of the various algorithms using as examples models of a structured Daphnia population interacting with an unstructured algal population. We use specifications of vital rates in terms of parameters and parameter values as in de Roos et al. (1990) and de Roos (1997). We have implemented the algorithms to compute existence and stability boundaries by numerical integration combined with curve tracing for the Daphnia model in a C-code. The parts of this code that deal with numerical integration and curve tracing are problem independent, whereas in other parts of the code the ingredients specific for the Daphnia model are used. We present results of the computations for the Daphnia model in the form of numerical examples and graphs of traced curves in two-parameter planes. We tested our algorithms by comparing our results with stability curves computed following the approaches in de Roos et al. (1990) and de Roos (1997), in which curves were traced after deriving analytic expressions for the integrals over the vital rates for the Daphnia model. The curves we compute in this paper match with the curves computed in de Roos et al. (1990) and de Roos (1997). 


\section{Consumer resource dynamics}

We present the analytic formulation of the class of models here that we will investigate. We first give the population equations and equilibrium conditions. Then we define existence and stability boundaries in two parameter planes. Much of Sections 2.1 and 2.2 overlap with de Roos et al. (2009) and is presented here for the sake of completeness.

\subsection{The model}

We first derive the population equations - stepping from the individual level to the population level-formally, i.e., we postpone smoothness discussions. We then give smoothness assumptions, discuss discontinuities induced by an abrupt onset of reproduction, and finally become more specific about the derivation of size and survival as functions of age.

\subsubsection{The population equations formally}

Let us denote by $S(t)$ the available resource or food concentration at time $t$. Our way of bookkeeping at the population level leads us to introduce histories, first as functions defined on $(-\infty, 0]$. For the resource $S$, we introduce the notation

$$
S_{t}(\sigma):=S(t+\sigma), \quad \sigma \in(-\infty, 0],
$$

which is common in the theory of functional differential equations (Hale, 1977). Then $S_{t}$ is a history for every $t$, the history of the resource at time $t$.

Let us assume that there is only one possible size $x_{b}$ at which individuals are born. Next, we introduce $X(a, \Psi)$ as the size that an individual has at age $a$, given that it has experienced history $\Psi$ in the time interval $[-a, 0]$. Then $X\left(a, S_{t}\right)$ is the size that an individual has at age $a$ and time $t$, given that it has experienced resource concentration $S$ in the time interval $[t-a, t]$; see Fig. 1. Likewise, we introduce $\mathcal{F}(a, \Psi)$ as the survival probability to age $a$ of an individual, given that if it survives, it has experienced history $\Psi$ in the time interval $[-a, 0]$. Then $\mathcal{F}\left(a, S_{t}\right)$ is the probability for an individual to reach age $a$ at time $t$ given that it experiences resource concentration $S$ in the time interval $[t-a, t]$. Next, we denote by $\beta(x, y)$ the rate of reproduction of an individual of size $x$ under resource condition $y$ and by $\gamma(x, y)$ the rate of food consumption of an individual of size $x$ under resource condition $y$. So, for example, $\beta\left(X\left(a, S_{t}\right), S(t)\right)$ is the rate of reproduction of an individual of age $a$ at time $t$. Finally, $f(y)$ denotes the intrinsic rate of change of the resource, meaning the rate of change in absence of the consumer. Let us denote by $b(t)$ the population birth rate, i.e., the number of individuals born (in total or per unit of area or volume, depending on the context) with size $x_{b}$ per unit of time at time $t$. Let us denote by $h>0$ the maximum lifetime of an individual under ideal food conditions. Then we can describe the population dynamics by the system of equations

$$
\begin{aligned}
b(t) & =\int_{0}^{h} \beta\left(X\left(a, S_{t}\right), S(t)\right) \mathcal{F}\left(a, S_{t}\right) b(t-a) d a, \\
S^{\prime}(t) & =f(S(t))-\int_{0}^{h} \gamma\left(X\left(a, S_{t}\right), S(t)\right) \mathcal{F}\left(a, S_{t}\right) b(t-a) d a ;
\end{aligned}
$$

see again Fig. 1. The Eqs. (2)-(3) form a system of a renewal equation (2) coupled to a delay differential equation (3). The qualitative behavior of such systems can be studied using the theory developed in Diekmann et al. (1995, 2007), and de Roos et al. (2009). 
contribution to births

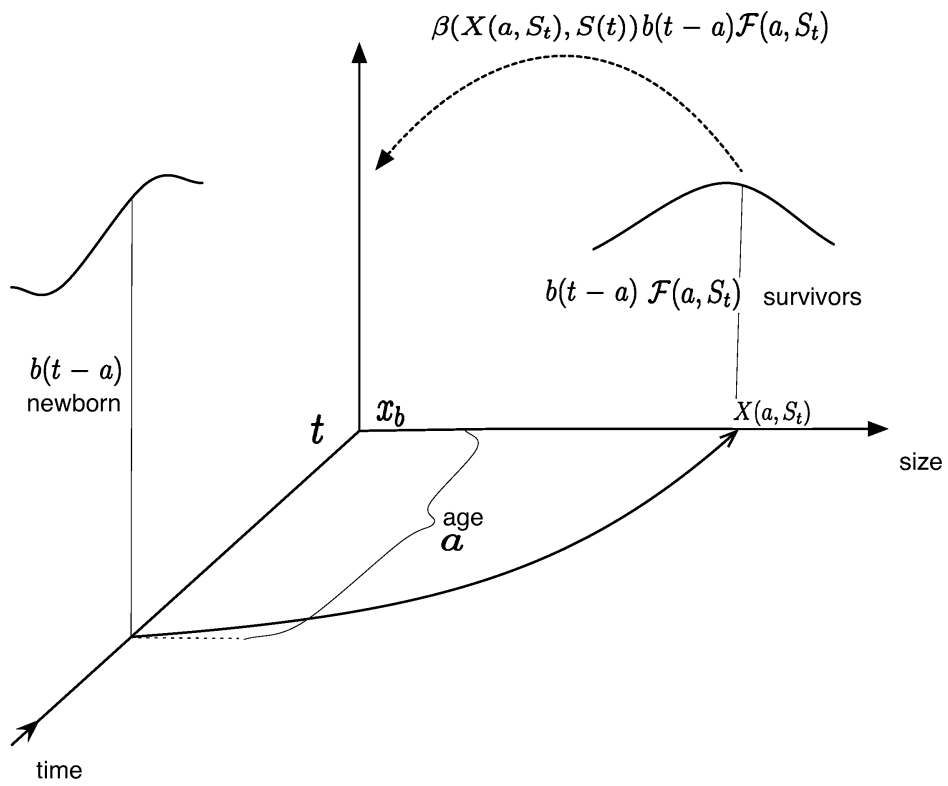

Fig. 1 Equation (2): reproduction at time $t$ as induced by the history of resource and population birth rate.

\subsubsection{Smoothness conditions and discontinuities caused by abrupt onset of reproduction} Motivated by the "Daphnia consuming algae" model in de Roos et al. (1990), we suppose that at size $x_{A}>x_{b}$ juveniles mature. Keeping in mind that we would like to linearize later, we should formalize the assumption that rates are smooth, except for a possible jump in $x_{A}$. We thus assume that $\beta$ and $\gamma$ can be defined on $\left[x_{b}, \infty\right)$ via functions that are $C^{1}$ on $\left[x_{b}, x_{A}\right] \times \mathbb{R}_{+}$and on $\left[x_{A}, \infty\right) \times \mathbb{R}_{+}$. This leads to unique definitions on $\left[x_{b}, \infty\right) \backslash\left\{x_{A}\right\} \times$ $\mathbb{R}_{+}$and to a double definition in $\left(x_{A}, y\right)$ for all $y \in \mathbb{R}_{+}$. This double definition, however, is irrelevant, as we are ultimately interested in the integrated functions. Finally, we assume that $\beta(x, y)=0$ for all $x \in\left[x_{b}, x_{A}\right)$ and all $y$. So, neither for $\beta$, nor for $\gamma$ there can be expected continuity, let alone differentiability, in $x_{A}$. Next, suppose that $f: \mathbb{R}_{+} \longrightarrow \mathbb{R}$ is $C^{1}$. We assume that $S$ is continuous and restrict the domain of definition of histories, in particular of $S_{t}$, from $(-\infty, 0]$ to $[-h, 0]$. To guarantee integrability in (2)-(3) and for later differentiability in the $S$-component, we require that $X$ and $\mathcal{F}$ are such that

$$
\begin{aligned}
C([-h, 0], \mathbb{R}) & \longrightarrow L^{\infty}([0, h], \mathbb{R}), \\
\psi & \longmapsto X(\cdot, \psi), \\
\psi & \longmapsto \mathcal{F}(\cdot, \psi)
\end{aligned}
$$

are continuously differentiable maps. Next, if an individual matures exactly at the present time for a given food history $\psi$, we denote its age at maturation by $a_{A}$, i.e., we define $a_{A}$ via the equation

$$
X\left(a_{A}, \psi\right)=x_{A},
$$


the solvability of which will be discussed in Section 2.1.3 below. Finally, we assume that $b$ is nonnegative and integrable. The population equations, reflecting the jump in $x_{A}$ are now given by

$$
\begin{aligned}
b(t)= & \int_{a_{A}\left(S_{t}\right)}^{h} \beta\left(X\left(a, S_{t}\right), S(t)\right) \mathcal{F}\left(a, S_{t}\right) b(t-a) d a, \\
S^{\prime}(t)= & f(S(t))-\int_{0}^{a_{A}\left(S_{t}\right)} \gamma\left(X\left(a, S_{t}\right), S(t)\right) \mathcal{F}\left(a, S_{t}\right) b(t-a) d a \\
& -\int_{a_{A}\left(S_{t}\right)}^{h} \gamma\left(X\left(a, S_{t}\right), S(t)\right) \mathcal{F}\left(a, S_{t}\right) b(t-a) d a,
\end{aligned}
$$

where the integrals from $a_{A}\left(S_{t}\right)$ to $h$ should be interpreted as zero when $a_{A}\left(S_{t}\right) \geq h$. In (7), we have split the integration interval into two parts in order to highlight the discontinuity in the integrand. In the following, however, we shall simply write one integral from zero to $h$, as the jump discontinuity is harmless with respect to integration.

\subsubsection{Computation of size and survival functions as solutions of $O D E$}

We show how $X$ and $\mathcal{F}$ can be computed for the case that one has given rates $g(x, y)$ and $\mu(x, y)$ of individual growth and mortality. Just like for $\gamma$, we also assume that $g, \mu: \mathbb{R}_{+}^{2} \longrightarrow \mathbb{R}_{+}$are $C^{1}$ on $\left[x_{b}, x_{A}\right] \times \mathbb{R}_{+}$and on $\left[x_{A}, \infty\right) \times \mathbb{R}_{+}$. We additionally assume the existence of positive lower bounds for $g$ and $\mu$ that are uniform for all sizes and uniform for all resource conditions outside a neighborhood of zero which should be chosen sufficiently small not to contain positive equilibria (which will be defined below).

The definition of $X$ and $\mathcal{F}$ in terms of the history of a time dependent resource via the rates $g$ and $\mu$ leads to a certain notational complexity. We here use the same notation as in de Roos et al. (2009). We denote by $x(\alpha)=x(\alpha ; a, \psi)$ the size of an individual at age $\alpha$, given that at age $a$, if still alive, it has experienced resource history $\psi$. Likewise, we denote by $\tilde{\mathcal{F}}(\alpha)=\tilde{\mathcal{F}}(\alpha ; a, \psi)$ the probability that an individual survives up to age $\alpha$, given that at age $a$, if still alive, it has experienced resource history $\psi$. Then we can define

$$
X\left(a, S_{t}\right):=x\left(a ; a, S_{t}\right) \quad \text { and } \quad \mathcal{F}\left(a, S_{t}\right):=\tilde{\mathcal{F}}\left(a ; a, S_{t}\right)
$$

We compute $x(\alpha)$ and $\tilde{\mathcal{F}}(\alpha)$ by solving the system of (one-sidedly) coupled nonautonomous ODE

$$
\begin{aligned}
x^{\prime}(\alpha) & =g(x(\alpha), \psi(-a+\alpha)), \quad 0<\alpha \leq a, \\
x(0) & =x_{b}, \\
\tilde{\mathcal{F}}^{\prime}(\alpha) & =-\mu(x(\alpha), \psi(-a+\alpha)) \tilde{\mathcal{F}}(\alpha), \quad 0<\alpha \leq a, \\
\tilde{\mathcal{F}}(0) & =1 .
\end{aligned}
$$

Equations (8)-(9) are linearized with respect to $\psi$ in Appendix A to compute expressions for the derivatives of $X$ and $\mathcal{F}$ with respect to $\psi$. 


\subsection{Steady states}

For the system (6)-(7), an equilibrium is a pair of constants $(\bar{b}, \bar{S})$, such that $(b, S):=$ $(\bar{b}, \bar{S})$ fulfills (6)-(7). If $\bar{b}=0, \bar{S}$ should be such that $f(\bar{S})=0$. In this case, we have a trivial equilibrium $(0, \bar{S})$, which we disregard here. A nontrivial equilibrium is given by a pair of constants $(\bar{b}, \bar{S})$ fulfilling

$$
\begin{aligned}
& R_{0}(\bar{S})-1=0, \\
& f(\bar{S})-\Theta(\bar{S}) \bar{b}=0, \\
& R_{0}(\bar{S}):=\int_{\bar{\tau}}^{h} \beta(X(a, \bar{S}), \bar{S}) \mathcal{F}(a, \bar{S}) d a, \\
& \Theta(\bar{S}):=\int_{0}^{h} \gamma(X(a, \bar{S}), \bar{S}) \mathcal{F}(a, \bar{S}) d a,
\end{aligned}
$$

where we denote by

$$
\bar{\tau}:=a_{A}(\bar{S})
$$

the age at which individuals mature under steady state conditions. Note that $R_{0}(\bar{S})$ and $\Theta(\bar{S})$ are, respectively, the expected lifetime offspring production and the expected lifetime resource consumption of a consumer individual, which gives obvious interpretations of the steady state conditions. As, for $\Theta(\bar{S})>0,(11)$ can be solved explicitly with respect to $\bar{b}$, we write

$$
\bar{b}(\bar{S}):=\frac{f(\bar{S})}{\Theta(\bar{S})},
$$

and reduce the steady state problem to finding an $\bar{S}$ satisfying (10). A typical case is that (10) has a unique solution; see, e.g., de Roos et al. (1990), and also here we assume that this holds. We give a method to approximate the solution $\bar{S}$ of (10) in Section 3.2.

\subsubsection{Existence boundaries}

We suppose in the following that two model parameters, which we denote by $\alpha_{1}$ and $\alpha_{2}$, are free. We will or will not incorporate (free) parameter dependence of functions into the notation according to convenience and relevance in the context. We then rewrite (10)-(11) as

$$
\begin{aligned}
& R_{0}\left(\alpha_{1}, \alpha_{2}, \bar{S}\right)-1=0, \\
& \bar{b}:=\frac{f\left(\alpha_{1}, \alpha_{2}, \bar{S}\right)}{\Theta\left(\alpha_{1}, \alpha_{2}, \bar{S}\right)},
\end{aligned}
$$

(where it will prove convenient in Section 3 to denote first parameters and then the equilibrium). The $\bar{b}$ component of the equilibrium becomes positive if the curve in the $\alpha_{1}-\alpha_{2}$-plane defined by the two equations

$$
f\left(\alpha_{1}, \alpha_{2}, \bar{S}\right)=0
$$


and (14) is crossed in the appropriate sense. We hence call this curve the existence boundary for the nontrivial equilibrium.

\subsubsection{Existence boundaries for Daphnia models and equilibrium curves}

In the models of Daphnia consuming algae, we assume (in absence of Daphnia) chemostat or logistic algal dynamics and choose $\alpha_{1}$ as the mortality for Daphnia and $\alpha_{2}$ as the carrying capacity for algae. Hence, typically, for given $\bar{S}, f$ is independent of $\alpha_{1}$ and $R_{0}$ independent of $\alpha_{2}$. Moreover, (16) is equivalent to $\bar{S}=\alpha_{2}$ for chemostat or to " $\bar{S}=0$ or $\bar{S}=\alpha_{2}$ " for logistic dynamics; see Section 4 below. Finally, typically (14) has no solution for $\bar{S}=0$, as in the absence of food there is no reproduction. In summary, for Daphnia, the existence boundary defined by (14) and (16) can equivalently be defined by

$$
\bar{S}=\alpha_{2}, \quad R_{0}\left(\alpha_{1}, \bar{S}\right)-1=0,
$$

where we dropped the nonmanifesting $\alpha_{2}$-dependence in the notation of $R_{0}$. It is hence clear that in this special setting the existence boundary in the $\alpha_{1}-\alpha_{2}$-plane and the curve describing how the equilibrium changes if one parameter varies are equal. We will give methods to compute existence boundaries for both the general setting and the setting for Daphnia in Section 3.

\subsubsection{Steady state defining functions as $O D E$ and stopping criteria for integration}

As in Kirkilionis et al. (2001), in view of the interdependence of functions and the discontinuity at $x_{A}$ we propose to compute several model ingredients as solutions of ODE. We introduce two alternative criteria for ending the integration of the ODE. The choice of which one to use should depend on the biological problem one considers. The first is the reaching of a maximum age $A_{\max }$, in which case we should choose $h:=A_{\max }$. The second criterion is the one proposed in Kirkilionis et al. (2001): For every $\varepsilon \in(0,1)$, we define $a_{\varepsilon}=a_{\varepsilon}(\bar{S})$ via the equation $\mathcal{F}\left(a_{\varepsilon}, \bar{S}\right)=\varepsilon$, i.e., as the age at which the probability that an individual survives up to it has decreased to $\varepsilon$, and assume that the function $\bar{S} \mapsto a_{\varepsilon}(\bar{S})$ is bounded, which is granted, if $\mathcal{F}$ is defined via $\mu$ as in Section 2.1.3. We call $\varepsilon$ the survival tolerance. When using this stopping criterium, we redefine $h$ as the supremum of the function $\bar{S} \mapsto a_{\varepsilon}(\bar{S})$. To facilitate the exposition, we will here and in the algorithms below concentrate on the second criterion, also since we believe that it is obvious what should be changed when using the first. We have implemented both criteria and will show the results for both. We associate with the steady state conditions the system of coupled autonomous ODE

$$
\begin{aligned}
\frac{d}{d a} X(a, \bar{S}) & =g(X(a, \bar{S}), \bar{S}), \quad a>a_{0}, \\
X\left(a_{0}, \bar{S}\right) & =x_{0}, \\
\frac{d}{d a} \mathcal{F}(a, \bar{S}) & =-\mu(X(a, \bar{S}), \bar{S}) \mathcal{F}(a, \bar{S}), \quad a>a_{0}, \\
\mathcal{F}\left(a_{0}, \bar{S}\right) & =f_{0}, \\
\frac{d}{d a} r(a, \bar{S}) & =\beta(X(a, \bar{S}), \bar{S}) \mathcal{F}(a, \bar{S}), \quad a>a_{0}, \\
r\left(a_{0}, \bar{S}\right) & =r_{0},
\end{aligned}
$$




$$
\begin{aligned}
\frac{d}{d a} \theta(a, \bar{S}) & =\gamma(X(a, \bar{S}), \bar{S}) \mathcal{F}(a, \bar{S}), \quad a>a_{0}, \\
\theta\left(a_{0}, \bar{S}\right) & =\theta_{0} .
\end{aligned}
$$

Note that $r$ denotes cumulative offspring, so corresponds to $R_{0}$ and $\theta$ cumulative consumption, so corresponds to $\Theta$. We have written the ODE for arbitrary initial data, as we would like to be able to solve the ODE for various initial data to cope with discontinuities. The natural start is with $a_{0}=0, x_{0}=x_{b}, f_{0}=1, r_{0}=0$, and $\theta_{0}=0$.

Remark 2.1. Of course $X$ and $\mathcal{F}$ as defined by (8)-(9) equal $X$ and $\mathcal{F}$ as defined by (18)(19) only for these natural initial conditions. Since we use the two sets of ODE in different contexts, this should not lead to misunderstanding.

Then if $a_{0}=0, x_{0}=x_{b}, f_{0}=1, r_{0}=0$, and $\theta_{0}=0$, we can integrate from zero to $a_{A}$ and next, with initial conditions guaranteeing continuity, from $a_{A}$ to $h$ to get $R_{0}(\bar{S})=r(h, \bar{S})$ and $\Theta(\bar{S})=\theta(h, \bar{S})$. We therefore introduce $R_{\varepsilon}(\bar{S}):=r\left(a_{\varepsilon}, \bar{S}\right)($ consistent for $\varepsilon=0), \Theta_{\varepsilon}(\bar{S}):=\theta\left(a_{\varepsilon}, \bar{S}\right)$ and $\bar{b}_{\varepsilon}(\bar{S}):=\frac{f(\bar{S})}{\Theta_{\varepsilon}(\bar{S})}$ as approximations for $R_{0}(\bar{S}), \Theta(\bar{S})$ and $\bar{b}(\bar{S})$.

\subsubsection{Definition of approximated existence boundaries}

For general consumer resource models, the approximated existence boundary for equilibria is the curve defined by

$$
G_{\varepsilon}\left(\alpha_{1}, \alpha_{2}, \bar{S}\right)=0
$$

where

$$
G_{\varepsilon}\left(\alpha_{1}, \alpha_{2}, \bar{S}\right):=\left(\begin{array}{c}
R_{\varepsilon}\left(\alpha_{1}, \alpha_{2}, \bar{S}\right)-1 \\
f\left(\alpha_{1}, \alpha_{2}, \bar{S}\right)
\end{array}\right)
$$

and where $R_{\varepsilon}$ can be computed via integration of (18)-(20). For Daphnia models, the existence boundary can be computed by tracing the curve defined by

$$
G_{\varepsilon}\left(\alpha_{1}, \bar{S}\right)=0
$$

where

$$
G_{\varepsilon}\left(\alpha_{1}, \bar{S}\right):=R_{\varepsilon}\left(\alpha_{1}, \bar{S}\right)-1,
$$

and where again $R_{\varepsilon}$ can be computed via integration of (18)-(20).

\subsection{Stability boundaries in a two parameter plane}

By the principle of linearized stability, see Diekmann et al. (2007) where this result is proven for (6)-(7); an equilibrium is locally stable, if all roots of the characteristic equation have negative real part and unstable if at least one root has positive real part. Hence, a necessary condition for a stability switch upon parameter variation is that at least one root 
crosses the imaginary axis (recall that non-real roots cross in complex conjugate pairs). As a first step, we thus derive the characteristic equation for (6)-(7). In the case of two free parameters, one can then define stability boundaries for equilibria in $\mathbb{R}^{2}$, interpreted as $\alpha_{1}-\alpha_{2}$-space, as explained below.

\subsubsection{The characteristic equation}

We will show in Appendix A that if one linearizes (6)-(7) and plugs in exponential trial solutions, one gets a characteristic equation of the form

$$
\operatorname{det}\left(M(\bar{b}, \bar{S}, \lambda)-\left(\begin{array}{ll}
1 & 0 \\
0 & \lambda
\end{array}\right)\right)=0,
$$

where, if one uses that (6)-(7) are linear in $\bar{b}$ for given $\bar{S}$, one can show that $M$ is of the form

$$
M(\bar{b}, \bar{S}, \lambda)=\left(\begin{array}{cc}
\Psi^{1}(\bar{S}, \lambda) & \bar{b} \Psi^{2}(\bar{S}, \lambda) \\
\Psi^{3}(\bar{S}, \lambda) & f^{\prime}(\bar{S})+\bar{b} \Psi^{4}(\bar{S}, \lambda)
\end{array}\right),
$$

for functions $\Psi^{i}, i=1, \ldots, 4$ to be specified (where $i$ is a super-index, not a power). The $\lambda$-dependence in $M$ is induced by the delay and to understand the form of the subtracted matrix recall that (2)-(3) is a renewal equation and a delay differential equation, such that the $\lambda$ arises through differentiation. We refer to (de Roos et al., 2009) for a representation involving Laplace transforms, which for numerical computations is less convenient. If we plug (13) into (27), we see that (26) is equivalent to

$$
\begin{aligned}
0= & f(\bar{S})\left[\left(\Psi^{1}(\bar{S}, \lambda)-1\right) \Psi^{4}(\bar{S}, \lambda)-\Psi^{2}(\bar{S}, \lambda) \Psi^{3}(\bar{S}, \lambda)\right] \\
& +\Theta(\bar{S})\left(\Psi^{1}(\bar{S}, \lambda)-1\right)\left(f^{\prime}(\bar{S})-\lambda\right) .
\end{aligned}
$$

\subsubsection{Stability boundaries}

To define stability boundaries, we should first plug $\lambda=i \omega, \omega \geq 0$, into (28) and rewrite the complex equation as two real equations. To this aim, we define real functions $\Psi_{r}^{j}(\bar{S}, \omega)$, $\Psi_{i}^{j}(\bar{S}, \omega), j=1, \ldots, 4$, such that

$$
\Psi^{j}(\bar{S}, i \omega)=\Psi_{r}^{j}(\bar{S}, \omega)+i \Psi_{i}^{j}(\bar{S}, \omega)
$$

(and where the subindices $r$ and $i$ refer to "real" and "imaginary"). If we plug this into (28), a straightforward computation shows that the complex equation (28) is equivalent to the system of two real equations defined by

$$
H(\bar{S}, \omega)=0,
$$

where

$$
\begin{aligned}
H(\bar{S}, \omega):= & f(\bar{S})\left[\left(\Psi_{r}^{1}(\bar{S}, \omega)-1\right)\left(\begin{array}{c}
\Psi_{r}^{4}(\bar{S}, \omega) \\
\Psi_{i}^{4}(\bar{S}, \omega)
\end{array}\right)+\Psi_{i}^{1}(\bar{S}, \omega)\left(\begin{array}{c}
-\Psi_{i}^{4}(\bar{S}, \omega) \\
\Psi_{r}^{4}(\bar{S}, \omega)
\end{array}\right)\right. \\
& \left.-\Psi_{r}^{3}(\bar{S}, \omega)\left(\begin{array}{l}
\Psi_{r}^{2}(\bar{S}, \omega) \\
\Psi_{i}^{2}(\bar{S}, \omega)
\end{array}\right)+\Psi_{i}^{3}(\bar{S}, \omega)\left(\begin{array}{c}
\Psi_{i}^{2}(\bar{S}, \omega) \\
-\Psi_{r}^{2}(\bar{S}, \omega)
\end{array}\right)\right]
\end{aligned}
$$




$$
+\Theta(\bar{S})\left[f^{\prime}(\bar{S})\left(\begin{array}{c}
\Psi_{r}^{1}(\bar{S}, \omega)-1 \\
\Psi_{i}^{1}(\bar{S}, \omega)
\end{array}\right)+\omega\left(\begin{array}{c}
\Psi_{i}^{1}(\bar{S}, \omega) \\
1-\Psi_{r}^{1}(\bar{S}, \omega)
\end{array}\right)\right]
$$

If we now incorporate two parameters $\alpha_{1}$ and $\alpha_{2}$ into the notation, we can define a system of three real equations

$$
\begin{aligned}
& G\left(\alpha_{1}, \alpha_{2}, \bar{S}\right)=0, \\
& H\left(\alpha_{1}, \alpha_{2}, \bar{S}, \omega\right)=0,
\end{aligned}
$$

where

$$
G\left(\alpha_{1}, \alpha_{2}, \bar{S}\right):=R_{0}\left(\alpha_{1}, \alpha_{2}, \bar{S}\right)-1,
$$

in the four variables $\bar{S}, \omega, \alpha_{1}$, and $\alpha_{2}$. Hence, generically (32) defines a curve that can be projected to the $\alpha_{1}-\alpha_{2}$-plane.

Remark 2.2. Note that for $p \geq 2$ in $p$-dimensional parameter space the stability boundary is of dimension $p-1$. We choose $p=2$ for the following reasons. For $p=2$, Eqs. (32) define a curve, for the numerical computation of which we can adapt established predictorcorrector algorithms. For $p=3$, the equations analogous to (32) would define a surface in three- dimensional parameter space, an object much more difficult to compute numerically than a curve. For $p \geq 4$, in addition to numerical difficulties that this choice would involve, the shape of the stability boundary would be hard to understand due to constraints of the human visual system.

We call the region in parameter space, where the positive equilibrium is stable, the stability region. The stability boundary is the boundary of this region. The stability region may have several components. Even if the stability region consists of one component, the boundary may consist of several connected components. We determine these components as solutions of (32) with either $\omega=0$ or $\omega>0$. (Note that for $\omega=0$ system (32) reduces to two equations in the three variables $\bar{S}, \alpha_{1}$, and $\alpha_{2}$, since the component of $H$ corresponding to the imaginary part is identically zero for $\omega=0$.)

In order that a solution of (32) corresponds to a component of the stability boundary, the number of roots of the characteristic equation in the right-half plane should be zero on one side of the curve. In the present case, there is only one curve corresponding to $\omega=0$ and this is the already computed existence boundary. At the existence boundary, the trivial and the nontrivial equilibrium exchange stability (we call this a "supercritical" transcritical bifurcation, in order to express that the nontrivial equilibrium is positive, and hence biologically meaningful, for parameter values for which the trivial steady state is unstable). So, from now on, we focus on $\omega>0$. Since roots occur in complex conjugate pairs, solution curves of (32) now correspond to Hopf bifurcation (if we cross the curve transversally in a regular point; note that, for instance, we may have two pairs crossing the imaginary axis in a self-intersection of the curve; see, e.g., Fig. 5 below).

Remark 2.3. Note that there may be other curves at which roots are on the imaginary axis and such that Hopf bifurcations occur, but which are not a stability boundary, since there 
are roots in the right-half plane at both sides of the curve; see Chapter XI in Diekmann et al. (1995) (whether one speaks of one or several curves may depend on whether or not one includes the point at infinity in the parameter plane).

In Sections 3.5-3.6, we give methods to trace stability boundaries numerically.

\subsubsection{The entries of the characteristic matrix}

We here give representations for the functions $\Psi^{i}$ in (27). In steady state, the resource abundance is time independent and, therefore, so is the size-age relation. Since in addition the representation of the $\Psi^{j}$ is quite involved, we introduce the shorter notation

$$
\begin{aligned}
\beta(a) & :=\beta(X(a, \bar{S}), \bar{S}), \quad \beta_{1}(a):=\left.\frac{\partial}{\partial x} \beta(x, S)\right|_{(x, S)=(X(a, \bar{S}), \bar{S})}, \\
\beta_{2}(a) & :=\left.\frac{\partial}{\partial S} \beta(x, S)\right|_{(x, S)=(X(a, \bar{S}), \bar{S})}, \quad \mathcal{F}(a):=\mathcal{F}(a, \bar{S})
\end{aligned}
$$

and analogously for $g_{i}, \gamma_{i}$, and $\mu_{i}$, where $i=1,2$. It is important to keep in mind, however, the dependence of functions, rates, and derivatives on the steady state condition $\bar{S}$, on the function $X(a, \bar{S})$, which is given only implicitly, and on parameters. In Appendix A, we derive (26) and from it that

$$
\begin{aligned}
\Psi^{1}(\bar{S}, \lambda)= & \int_{\bar{\tau}}^{h} e^{-\lambda a} \beta(a) \mathcal{F}(a) d a \\
\Psi^{3}(\bar{S}, \lambda)= & -\int_{0}^{h} e^{-\lambda a} \gamma(a) \mathcal{F}(a) d a \\
\Psi^{2}(\bar{S}, \lambda)= & \int_{\bar{\tau}}^{h} \beta_{2}(a) \mathcal{F}(a) d a \\
& +\int_{\bar{\tau}}^{h} e^{-\lambda a}\left[\beta(a) L(a, \lambda)+\beta_{1}(a) \mathcal{F}(a) K(a, \lambda)\right] d a \\
& +\left(\frac{g^{+}}{g^{-}}-1\right) e^{-\lambda \bar{\tau}} K(\bar{\tau}, \lambda) \int_{\bar{\tau}}^{h} \beta_{1}(\sigma) \mathcal{F}(\sigma) d \sigma \\
& +\frac{\mu^{-}-\mu^{+}}{g^{-}} K(\bar{\tau}, \lambda) \int_{\bar{\tau}}^{h} \beta(a) \mathcal{F}(a) e^{-\lambda a} d a \\
& +\frac{\mathcal{F}(\bar{\tau}) \beta^{+}}{g^{-}} e^{-\lambda \bar{\tau}} K(\bar{\tau}, \lambda), \\
& -\left(\frac{g^{+}}{g^{-}}-1\right) e^{-\lambda \bar{\tau}} K(\bar{\tau}, \lambda) \int_{\bar{\tau}}^{h} \gamma_{1}(\sigma) \mathcal{F}(\sigma) d \sigma \\
& \left.-\int_{0}^{h} e^{-\lambda a}\left[\gamma(a) L(a, \lambda)+\gamma_{1}(a) \mathcal{F}(a) K(a) d a\right)\right] d a \\
\Psi^{4}(\bar{S}, \lambda)= &
\end{aligned}
$$




$$
\begin{aligned}
& -\frac{\mu^{-}-\mu^{+}}{g^{-}} K(\bar{\tau}, \lambda) \int_{\bar{\tau}}^{h} \gamma(a) \mathcal{F}(a) e^{-\lambda a} d a \\
& -\frac{\mathcal{F}(\bar{\tau})}{g^{-}}\left[\gamma^{+}-\gamma^{-}\right] e^{-\lambda \bar{\tau}} K(\bar{\tau}, \lambda),
\end{aligned}
$$

defining $g^{+}:=g\left(x_{A}+, \bar{S}\right), g^{-}:=g\left(x_{A}-, \bar{S}\right)$ as the one-sided limits, analogously for the other rates, and $K$ and $L$ as the solutions of

$$
\begin{aligned}
\frac{\partial}{\partial a} K(a, \lambda) & =g_{1}(a) K(a, \lambda)+g_{2}(a) e^{\lambda a}, \quad a>a_{0} \\
K\left(a_{0}, \lambda\right) & =k^{0} \\
\frac{\partial}{\partial a} L(a, \lambda) & =-\mu(a) L(a, \lambda)-\mu_{1}(a) K(a, \lambda) \mathcal{F}(a)-\mu_{2}(a) e^{\lambda a} \mathcal{F}(a), \\
a>a_{0} & \\
L\left(a_{0}, \lambda\right) & =l^{0}
\end{aligned}
$$

for $a_{0}:=0, k^{0}:=0$ and $l^{0}:=0$.

Remark 2.4. Note that if all vital rates are additionally continuous in $x_{A}$ the last three terms in $\Psi^{2}$ and $\Psi^{4}$ equal zero (note that such a continuity assumption would imply that $\beta^{+}=0$ ), such that the situation simplifies considerably.

\subsubsection{Computing entries in the characteristic matrix by solving real ODE}

The definition of our curve involves the system of real equations (30) for the real functions $\Psi_{i}^{j}, \Psi_{r}^{j}, j=1, \ldots, 4$. These functions are defined via the complex functions $\Psi^{j}$, $j=1, \ldots, 4$ in (29), which are defined in (34)-(38). Moreover, the ODE-solver in our implementation requires the specification of real ODE. We therefore in the following redefine $\Psi_{i}^{j}(\bar{S}, \omega)$ and $\Psi_{r}^{j}(\bar{S}, \omega)$ as solutions of real ODE.

First, we plug $\lambda=i \omega$ into (37)-(38) and rewrite these equations as a system of ODE for real functions $K_{r}, K_{i}, L_{r}, L_{i}$, which fulfill (when we suppress the dependence on $\omega$ and $\bar{S}$ in the notation)

$$
K(a, i \omega)=K_{r}(a)+i K_{i}(a), \quad L(a, i \omega)=L_{r}(a)+i L_{i}(a)
$$

We then get

$$
\begin{aligned}
& K_{r}^{\prime}(a)=g_{1}(a) K_{r}(a)+g_{2}(a) \cos \omega a, \quad a>a_{0}, \\
& K_{i}^{\prime}(a)=g_{1}(a) K_{i}(a)+g_{2}(a) \sin \omega a, \\
& K_{r}\left(a_{0}\right)=k_{r}^{0}, \quad K_{i}\left(a_{0}\right)=k_{i}^{0}, \\
& L_{r}^{\prime}(a)=-\mu(a) L_{r}(a)-\mu_{1}(a) \mathcal{F}(a) K_{r}(a)-\mu_{2}(a) \mathcal{F}(a) \cos \omega a, \quad a>a_{0}, \\
& L_{i}^{\prime}(a)=-\mu(a) L_{i}(a)-\mu_{1}(a) \mathcal{F}(a) K_{i}(a)-\mu_{2}(a) \mathcal{F}(a) \sin \omega a, \quad \\
& L_{r}\left(a_{0}\right)=l_{r}^{0}, \quad L_{i}\left(a_{0}\right)=l_{i}^{0} .
\end{aligned}
$$


To write down the real ODE to redefine (34)-(36) for $\lambda=i \omega$ it is convenient to introduce real functions

$$
\begin{aligned}
& K_{r}^{e}\left(a_{1}, a_{2}\right):=\operatorname{Re}\left(e^{-i \omega a_{1}} K\left(a_{2}, i \omega\right)\right)=K_{r}\left(a_{2}\right) \cos \omega a_{1}+K_{i}\left(a_{2}\right) \sin \omega a_{1}, \\
& K_{i}^{e}\left(a_{1}, a_{2}\right):=\operatorname{Im}\left(e^{-i \omega a_{1}} K\left(a_{2}, i \omega\right)\right)=K_{i}\left(a_{2}\right) \cos \omega a_{1}-K_{r}\left(a_{2}\right) \sin \omega a_{1}
\end{aligned}
$$

and analogously $L_{r}^{e}\left(a_{1}, a_{2}\right)$ and $L_{i}^{e}\left(a_{1}, a_{2}\right)$. Next, we should define real functions $\psi_{r}^{j}=$ $\psi_{r}^{j}(a, \bar{S}, \omega)$ and $\psi_{i}^{j}=\psi_{i}^{j}(a, \bar{S}, \omega), j=1, \ldots, 4$, such that $\Psi_{r}^{j}(\bar{S}, \omega)=\psi_{r}^{j}(h, \bar{S}, \omega)$ and $\Psi_{i}^{j}(\bar{S}, \omega)=\psi_{i}^{j}(h, \bar{S}, \omega)$ with $\Psi_{r}^{j}$ and $\Psi_{i}^{j}$ as in Section 2.3.2. This holds if we define $\psi_{r}^{j}$ and $\psi_{i}^{j}$ via the real ODE

$$
\begin{aligned}
& \frac{d}{d a} \psi_{r}^{1}(a)=\beta(a) \mathcal{F}(a) \cos \omega a, \\
& \frac{d}{d a} \psi_{i}^{1}(a)=-\beta(a) \mathcal{F}(a) \sin \omega a, \quad a>a_{0}, \\
& \psi_{r}^{1}\left(a_{0}\right)=\psi_{1, r}^{0}, \quad \psi_{i}^{1}\left(a_{0}\right)=\psi_{1, i}^{0}, \\
& \frac{d}{d a} \psi_{r}^{2}(a)=\beta_{2}(a) \mathcal{F}(a)+\beta(a) L_{r}^{e}(a, a)+\beta_{1}(a) \mathcal{F}(a) K_{r}^{e}(a, a) \\
& +\left(\frac{g^{+}}{g^{-}}-1\right) \beta_{1}(a) \mathcal{F}(a) K_{r}^{e}(\bar{\tau}, \bar{\tau}) \\
& +\frac{\mu^{-}-\mu^{+}}{g^{-}} \beta(a) \mathcal{F}(a) K_{r}^{e}(a, \bar{\tau}), \\
& \frac{d}{d a} \psi_{i}^{2}(a)=\beta(a) L_{i}^{e}(a, a)+\beta_{1}(a) \mathcal{F}(a) K_{i}^{e}(a, a) \\
& +\left(\frac{g^{+}}{g^{-}}-1\right) \beta_{1}(a) \mathcal{F}(a) K_{i}^{e}(\bar{\tau}, \bar{\tau}) \\
& +\frac{\mu^{-}-\mu^{+}}{g^{-}} \beta(a) \mathcal{F}(a) K_{i}^{e}(a, \bar{\tau}), \quad a>a_{0}, \\
& \psi_{r}^{2}\left(a_{0}\right)=\psi_{2, r}^{0}, \quad \psi_{i}^{2}\left(a_{0}\right)=\psi_{2, i}^{0}, \\
& \frac{d}{d a} \psi_{r}^{3}(a)=-\gamma(a) \mathcal{F}(a) \cos \omega a, \\
& \frac{d}{d a} \psi_{i}^{3}(a)=\gamma(a) \mathcal{F}(a) \sin \omega a, \quad a>a_{0}, \\
& \psi_{r}^{3}\left(a_{0}\right)=\psi_{3, r}^{0}, \quad \psi_{i}^{3}\left(a_{0}\right)=\psi_{3, i}^{0}, \\
& \frac{d}{d a} \psi_{J, r}^{4}(a)=-\gamma_{2}(a) \mathcal{F}(a)-\gamma(a) L_{r}^{e}(a, a)-\gamma_{1}(a) \mathcal{F}(a) K_{r}^{e}(a, a), \\
& \frac{d}{d a} \psi_{J, i}^{4}(a)=-\gamma(a) L_{i}^{e}(a, a)-\gamma_{1}(a) \mathcal{F}(a) K_{i}^{e}(a, a), \\
& \psi_{J, r}^{4}\left(a_{0}\right)=\psi_{J, 4, r}^{0}, \quad \psi_{J, i}^{4}\left(a_{0}\right)=\psi_{J, 4, i}^{0},
\end{aligned}
$$$$
a>a_{0},(46)
$$ 


$$
\begin{aligned}
\frac{d}{d a} \psi_{r}^{4}(a)= & -\gamma_{2}(a) \mathcal{F}(a)-\gamma(a) L_{r}^{e}(a, a)-\gamma_{1}(a) \mathcal{F}(a) K_{r}^{e}(a, a) \\
& -\left(\frac{g^{+}}{g^{-}}-1\right) \gamma_{1}(a) \mathcal{F}(a) K_{r}^{e}(\bar{\tau}, \bar{\tau}) \\
& -\frac{\mu^{-}-\mu^{+}}{g^{-}} \gamma(a) \mathcal{F}(a) K_{r}^{e}(a, \bar{\tau}), \\
\frac{d}{d a} \psi_{i}^{4}(a)= & -\gamma(a) L_{i}^{e}(a, a)-\gamma_{1}(a) \mathcal{F}(a) K_{i}^{e}(a, a) \\
& -\left(\frac{g^{+}}{g^{-}}-1\right) \gamma_{1}(a) \mathcal{F}(a) K_{i}^{e}(\bar{\tau}, \bar{\tau}) \\
& -\frac{\mu^{-}-\mu^{+}}{g^{-}} \gamma(a) \mathcal{F}(a) K_{i}^{e}(a, \bar{\tau}), \\
a>a_{0}, \quad & \psi_{i}^{4}\left(a_{0}\right)=\psi_{4, i}^{0},
\end{aligned}
$$

for appropriate initial conditions, which will be specified in Sections 3.5-3.6 below. The functions $\psi_{J, r}^{4}$ and $\psi_{J, i}^{4}$ will be used to define initial conditions for $\psi_{r}^{4}$ and $\psi_{i}^{4}$ at $a_{\varepsilon}$.

\subsubsection{Definition of the approximated stability boundaries}

We define the approximation of $H$ as

$$
\begin{aligned}
H_{\varepsilon}(\bar{S}, \omega)= & f(\bar{S})\left[\left(\psi_{r}^{1}\left(a_{\varepsilon}, \bar{S}, \omega\right)-1\right)\left(\begin{array}{c}
\psi_{r}^{4}\left(a_{\varepsilon}, \bar{S}, \omega\right) \\
\psi_{i}^{4}\left(a_{\varepsilon}, \bar{S}, \omega\right)
\end{array}\right)\right. \\
& +\psi_{i}^{1}\left(a_{\varepsilon}, \bar{S}, \omega\right)\left(\begin{array}{c}
-\psi_{i}^{4}\left(a_{\varepsilon}, \bar{S}, \omega\right) \\
\psi_{r}^{4}\left(a_{\varepsilon}, \bar{S}, \omega\right)
\end{array}\right) \\
& \left.-\psi_{r}^{3}\left(a_{\varepsilon}, \bar{S}, \omega\right)\left(\begin{array}{c}
\psi_{r}^{2}\left(a_{\varepsilon}, \bar{S}, \omega\right) \\
\psi_{i}^{2}\left(a_{\varepsilon}, \bar{S}, \omega\right)
\end{array}\right)+\psi_{i}^{3}\left(a_{\varepsilon}, \bar{S}, \omega\right)\left(\begin{array}{c}
\psi_{i}^{2}\left(a_{\varepsilon}, \bar{S}, \omega\right) \\
-\psi_{r}^{2}\left(a_{\varepsilon}, \bar{S}, \omega\right)
\end{array}\right)\right] \\
& +\Theta(\bar{S})\left[f^{\prime}(\bar{S})\left(\begin{array}{c}
\psi_{r}^{1}\left(a_{\varepsilon}, \bar{S}, \omega\right)-1 \\
\psi_{i}^{1}\left(a_{\varepsilon}, \bar{S}, \omega\right)
\end{array}\right)+\omega\left(\begin{array}{c}
\psi_{i}^{1}\left(a_{\varepsilon}, \bar{S}, \omega\right) \\
1-\psi_{r}^{1}\left(a_{\varepsilon}, \bar{S}, \omega\right)
\end{array}\right)\right] .(48)
\end{aligned}
$$

Next, we incorporate dependence on $\alpha_{1}$ and $\alpha_{2}$ into the notation and define a map

$$
F_{\varepsilon}\left(\alpha_{1}, \alpha_{2}, \bar{S}, \omega\right):=\left(G_{\varepsilon}\left(\alpha_{1}, \alpha_{2}, \bar{S}\right), H_{\varepsilon}\left(\alpha_{1}, \alpha_{2}, \bar{S}, \omega\right)\right)
$$

where

$$
G_{\varepsilon}\left(\alpha_{1}, \alpha_{2}, \bar{S}\right):=R_{\varepsilon}\left(\alpha_{1}, \alpha_{2}, \bar{S}\right)-1 .
$$

We can then define the approximated stability boundary by

$$
F_{\varepsilon}\left(\alpha_{1}, \alpha_{2}, \bar{S}, \omega\right)=0
$$

where $F_{\varepsilon}$ is defined in (49) in terms of $G_{\varepsilon}$ and $H_{\varepsilon}$. The map $G_{\varepsilon}$ is defined in terms of $R_{\varepsilon}$ in (50), where $R_{\varepsilon}$ can be evaluated as described in Section 2.2.3. The map $H_{\varepsilon}$ is defined in (48) in terms of the functions $\psi_{r}^{j}$ and $\psi_{i}^{j}, j=1, \ldots, 4$ and $\Theta_{\varepsilon}$. The population birth 
rate $\bar{b}_{\varepsilon}$ is defined as

$$
\bar{b}_{\varepsilon}\left(\alpha_{1}, \alpha_{2}, \bar{S}\right)=\frac{f\left(\alpha_{1}, \alpha_{2}, \bar{S}\right)}{\Theta_{\varepsilon}\left(\alpha_{1}, \alpha_{2}, \bar{S}\right)} .
$$

The functions $\psi_{r}^{j}$ and $\psi_{i}^{j}$ can be computed via (41)-(47) in terms of $X$ and $\mathcal{F}$ and finally $X, \mathcal{F}$ and $\Theta_{\varepsilon}$ are defined via (18), (19), and (21).

\section{Numerical approximation techniques}

We here give iterative methods to approximate solutions for the problems discussed in the last section. As in Kirkilionis et al. (2001), we present the methods in the form of algorithms in pseudo-code established in Allgower and Georg (1990). This pseudo-code is designed to be understandable independent of whether the reader is familiar with particular coding languages.

\subsection{Integration to compute equilibria}

The following two algorithms can be used to perform the numerical integration to compute $G_{\varepsilon}$ and $\bar{b}_{\varepsilon}$ for given $\bar{S}$ and a given set of parameters. These computations are used to locate equilibria, to continue them as a function of one parameter, to compute stability boundaries, and to compute the population birth rate for any of the located equilibria. The first algorithm, i.e., the algorithm to evaluate $G_{\varepsilon}(\bar{S})$ for given $\bar{S}$ can also be used to test how close an initial guess is to an equilibrium point. As numerical computation of both $r\left(a_{\varepsilon}, \bar{S}\right)$ and $\theta\left(a_{\varepsilon}, \bar{S}\right)$ involves integrating (18)-(19), it may seem most efficient to integrate the four ODE (18)-(21) simultaneously. We here, however, split the integration procedures for the two ingredients for the following reason. When, e.g., searching for solutions of $R_{0}(\bar{S})-1=0$ through an iterative process, the numerical integration to compute $r\left(a_{\varepsilon}, \bar{S}\right)$ has to be repeated for different $\bar{S}$, but integration of (21) is only needed for the computation of $\bar{b}$ via (13). Numerical integration of (21) hence only has to be carried out once with the final approximation for $\bar{S}$. We assume that we are able to choose the survival tolerance $\varepsilon$ so small that under the relevant food condition $\bar{S}$ individuals reach size $x_{A}$ before the survival probability decreases to $\varepsilon$.

Remark 3.1. For the implementation of the algorithms, we point out that stopping at $a_{A}$ and at $a_{\varepsilon}$ requires the use of a special integration method such as DOPRI5 or DOPRI8 (we have used DOPRI5) that can handle event detections; see Hairer et al. (1987).

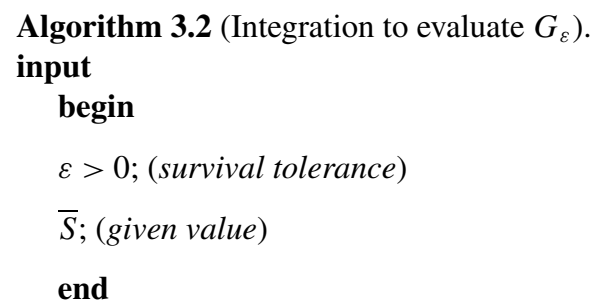


$a_{0}:=0, x_{0}:=x_{b}, f_{0}:=1$;

integrate in parallel (18)-(19) until $X(a, \bar{S})=x_{A}$;

set $\bar{\tau}:=a$;

store $\bar{\tau}$ and $\mathcal{F}(\bar{\tau}, \bar{S})$;

$a_{0}:=\bar{\tau}, x_{0}:=x_{A}, f_{0}:=\mathcal{F}(\bar{\tau}, \bar{S}), r_{0}:=0 ;$

integrate in parallel (18)-(19) and (20) until $\mathcal{F}(a, \bar{S})=\varepsilon$;

$a_{\varepsilon}:=a$

$G_{\varepsilon}(\bar{S}):=r\left(a_{\varepsilon}, \bar{S}\right)-1$.

Algorithm 3.3 (Computation of $\bar{b}_{\varepsilon}$ ).

input

begin

$\varepsilon>0 ;$ (survival tolerance)

$\bar{S} ;$ (given value)

end

$a_{0}:=0, x_{0}:=x_{b}, f_{0}:=1, \theta_{0}:=0 ;$

integrate in parallel (18)-(19) and (21) until $X(a, \bar{S})=x_{A}$;

set $\bar{\tau}:=a$;

store $\bar{\tau}, \mathcal{F}(\bar{\tau}, \bar{S})$ and $\theta(\bar{\tau}, \bar{S})$;

$a_{0}:=\bar{\tau}, x_{0}:=x_{A}, f_{0}:=\mathcal{F}(\bar{\tau}, \bar{S}), \theta_{0}:=\theta(\bar{\tau}, \bar{S}) ;$

integrate in parallel (18)-(19) and (21) until $\mathcal{F}(a, \bar{S})=\varepsilon$;

$a_{\varepsilon}:=a ;$

store $\theta\left(a_{\varepsilon}, \bar{S}\right)$;

compute $f(\bar{S})$;

$\bar{b}_{\varepsilon}:=\frac{f(\bar{S})}{\theta\left(a_{\varepsilon}, \bar{S}\right)}$.

\subsection{Approximation of equilibria}

If the set of parameters defining the vital rates is fixed, equilibria can be approximated by combining a Newton method with numerical integration. The Newton method involves the repeated calculation of the Jacobian, which in general is costly. To reduce these costs, we use Broyden updates of the Jacobian; see Allgower and Georg (1990) or Kuznetsov (1994). The use of Broyden updates requires one approximation of the Jacobian, which then can be updated with two successive curve points. 


\subsubsection{Numerical differentiation}

For our approximation of all partial derivatives, we use throughout two numerical constants $\delta_{1}$ and $\delta_{2}, \delta_{1}>\delta_{2}>0$. We then approximate the partial derivative of a function $F$ in $\bar{x}$ in the $i$ th component as

$$
\frac{\partial F}{\partial x_{i}}(\bar{x})=\frac{1}{\delta}\left(F\left(\bar{x}_{1}, \ldots, \bar{x}_{i}+\delta, \bar{x}_{i+1}, \ldots, \bar{x}_{N}\right)-F(\bar{x})\right),
$$

where $\delta:=\max \left\{\bar{x}_{i} \delta_{1}, \delta_{2}\right\}$. Here, the second argument in the maximum function is used when the point to be evaluated is close to zero.

\subsubsection{Broyden updates}

We denote by $A^{*}$ the transpose of a matrix $A$ and by $\|\cdot\|$ the Euclidean norm. Then we define the Broyden update of the Jacobian given two successive approximate solution points $y^{(k)}, y^{(k+1)}$ via

$$
\eta^{(k)}:=y^{(k+1)}-y^{(k)}, \quad \zeta^{(k)}:=G_{\varepsilon}\left(y^{(k+1)}\right)-G_{\varepsilon}\left(y^{(k)}\right),
$$

and

$$
A^{(k+1)}:=A^{(k)}+\frac{\left(\zeta^{(k)}-A^{(k)} \eta^{(k)}\right) \eta^{(k) *}}{\left\|\eta^{(k)}\right\|^{2}} .
$$

Algorithm 3.4 (Numerically solve $G_{\varepsilon}(\bar{S})=0$ ).

input

begin

$\varepsilon>0 ;($ survival tolerance)

$\delta_{1}, \delta_{2} ;($ accuracy constants for numerical differentiation)

$\varepsilon_{G}, \varepsilon_{y}>0 ;$ (numerical accuracy constants)

$\bar{S}^{(0)} ;$ (initial guess)

end

$y^{(0)}:=\bar{S}^{(0)}$

compute $G_{\varepsilon}\left(y^{(0)}\right)$ with Algorithm 3.2;

compute $A^{(0)} \approx G_{\varepsilon}^{\prime}\left(y^{(0)}\right)$ via numerical differentiation using Algorithm 3.2;

solve $A^{(0)} \eta^{(0)}=-G_{\varepsilon}\left(y^{(0)}\right)$ with respect to $\eta^{(0)}$;

$y^{(1)}:=y^{(0)}+\eta^{(0)} ;$ (new approximation to equilibrium)

compute $G_{\varepsilon}\left(y^{(1)}\right)$ with Algorithm 3.2;

if $\left\|\eta^{(0)}\right\|<\varepsilon_{y}$ and $\left\|G_{\varepsilon}\left(y^{(1)}\right)\right\|<\varepsilon_{G}$ then $\bar{S}:=y^{(1)}$; (algorithm stops)

else $k:=0 ;$ (start iteration with Broyden updates) 


\section{repeat (iteration loop)}

$k:=k+1$;

compute Broyden update $A^{(k)}$ with $y^{(k)}, y^{(k-1)}$ and (53);

solve $A^{(k)} \eta^{(k)}=-G_{\varepsilon}\left(y^{(k)}\right)$ with respect to $\eta^{(k)}$;

$y^{(k+1)}:=y^{(k)}+\eta^{(k)} ;$ (new approximation to equilibrium)

compute $G_{\varepsilon}\left(y^{(k+1)}\right)$ with Algorithm 3.2;

until

$$
\left\|\eta^{(k)}\right\|<\varepsilon_{y}, \quad\left\|G_{\varepsilon}\left(y^{(k+1)}\right)\right\|<\varepsilon_{G} ;
$$

$\bar{S}:=y^{(k+1)} ;$ (new approximation accepted $)$.

To compute an approximation of an equilibrium, it remains to combine the previous algorithm with the computation of the population birth rate via Algorithm 3.3 for the final value of $\bar{S}$.

Algorithm 3.5 (Approximation of equilibrium).

input

begin

$\varepsilon>0 ;$ (survival tolerance)

$\delta_{1}, \delta_{2} ;$ (accuracy constants for numerical differentiation)

$\varepsilon_{G}, \varepsilon_{y}>0 ;$ (numerical accuracy constants)

$\bar{S}^{(0)} ;($ starting point, initial guess)

end

find $\bar{S}$ numerically solving $G_{\varepsilon}(\bar{S})=0$ via Algorithm 3.4;

compute $\bar{b}_{\varepsilon}(\bar{S})$ via Algorithm 3.3;

$(\bar{b}, \bar{S}):=\left(\bar{b}_{\varepsilon}(\bar{S}), \bar{S}\right) ;($ approximation of the equilibrium $)$.

\subsection{Numerical tracing of curves}

We first outline the idea how to numerically trace a curve. We here use pseudo-arc length continuation as described in Kuznetsov (1994). We would like to numerically trace the curve defined by

$$
F(y)=0,
$$

where $F: \Omega \longrightarrow \mathbb{R}^{N}, \Omega \subset \mathbb{R}^{N+1}$. To apply the classical Newton method the number of equations should equal the number of unknowns, which is not the case in (54). To find 


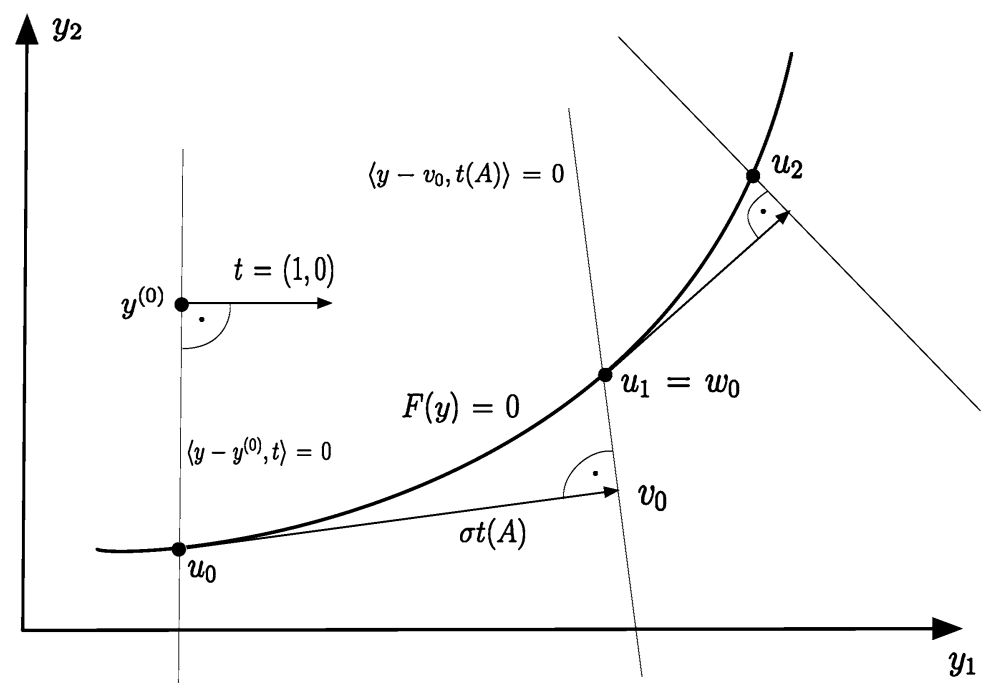

- Computations of the Jacobian

Fig. 2 Pseudo-arc length continuation.

a first point $y$ on the curve from an initial guess $y^{(0)}$, we can however simply fix one component, say $y_{1}^{(0)}$, and then test whether Algorithm 3.4 applied to the map

$$
\left(y_{2}, \ldots, y_{N+1}\right) \longmapsto F\left(y_{1}^{(0)}, y_{2}, \ldots, y_{N+1}\right)
$$

converges with initial guess $\left(y_{2}^{(0)}, \ldots, y_{N+1}^{(0)}\right)$. We will use the following equivalent alternative, however. Note that the solution to the above problem can equivalently be defined as the intersection of the curve with the hyperplane through $y^{(0)}$ and orthogonal to $(1,0, \ldots, 0)$; see Fig. 2 for the case $N=1$. This means numerically solving the problem

$$
\bar{F}(y)=0,
$$

where

$$
\bar{F}(y):=\left(F(y),\left\langle y-y^{(0)}, t\right\rangle\right)
$$

with $t:=(1,0, \ldots, 0)$ and starting point $y^{0}$. The advantage of using (55)-(56) is that we can reuse the algorithms to solve it for the computation of further curve points, as will become clear below.

Now, to continue the curve, once an initial solution point $u_{0}$ has been located, the next step is to predict a point $v_{0}$ by following the tangent $t(A), A=D F\left(u_{0}\right)$, to the curve in $u_{0}$ for a certain step-length $\sigma$, i.e., to set

$$
v_{0}=u_{0}+t(A) \sigma .
$$

We will explain below more precisely, how to define the normed and oriented tangent. 
To correct the predicted point, we define another Newton problem, now with starting point $v_{0}$. This time, however, we search for the intersection of the curve with the hyperplane through $v_{0}$ and orthogonal to the tangent $t(A)$ in $u_{0}$; see again Fig. 2. Hence, we try to numerically solve the problem defined by (55)-(56) with $t:=t(A)$ and $y^{(0)}:=v_{0}$. The solution $w_{0}$ to this problem is then the approximation of the second point on the curve.

It remains to define the normed and oriented tangent vector, which we do similarly as in Allgower and Georg (1990) and Kuznetsov (1994).

Definition 3.6. Let $A$ be an $n \times(n+1)$-matrix with $\operatorname{rank}(A)=n$. The unique vector $t=t(A) \in \mathbb{R}^{n+1}$ satisfying

(i) $A t=0$,

(ii) $\|t\|=1$,

(iii) $\operatorname{det}\left(\begin{array}{c}A \\ t^{*}\end{array}\right)>0$ is called the tangent vector induced by $A$.

If $A$ is the Jacobian in a curve point, then (i) results from the requirement of coincidence of the directions of curve and tangent vector in this point. Property (ii) says that the tangent should have norm one and (iii) that the orientation of the tangent should be preserved along the curve.

To compute a first point on the curve as well as to compute a correction for a predicted point, we use the following algorithm.

Algorithm 3.7 (Find intersection of curve and hyperplane).

input

begin

$F: \Omega \longrightarrow \mathbb{R}^{N}, \Omega \subset \mathbb{R}^{N+1} ;$ (map defining curve)

$y^{(0)} \in \mathbb{R}^{N+1} ;$ (initial guess)

evaluation algorithm for $F$;

$t \in \mathbb{R}^{N+1} ;$ (defining orthonormal vector for hyperplane)

$A \in \mathbb{R}^{N \times N} ;$ (approximation of $F^{\prime}\left(y^{(0)}\right)$ )

$\varepsilon_{y}, \varepsilon_{F}>0 ;$ (numerical accuracy constants)

end

define $\bar{F}(y):=\left(F(y),\left\langle y-y^{(0)}, t\right\rangle\right)$;

set $\bar{A}^{(0)}:=(A, t)$;

evaluate $F$ in $y^{(0)}$ and compute $\bar{F}\left(y^{(0)}\right)$;

solve $\bar{A}^{(0)} \eta^{(0)}=-\bar{F}\left(y^{(0)}\right)$ with respect to $\eta^{(0)}$;

$y^{(1)}:=y^{(0)}+\eta^{(0)} ;$ (new approximation to curve point)

evaluate $F$ in $y^{(1)}$ and compute $\bar{F}\left(y^{(1)}\right)$; 
if $\left\|\eta^{(0)}\right\|<\varepsilon_{y}$ and $\left\|\bar{F}\left(y^{(1)}\right)\right\|<\varepsilon_{F}$ then $u:=y^{(1)}$; (curve point found)

else $k:=0$ (start iteration with Broyden update)

\section{repeat}

$k:=k+1$

compute Broyden update $\bar{A}^{(k)}$ with $y^{(k)}, y^{(k-1)}, A^{(k-1)}$ and (53);

solve $\bar{A}^{(k)} \eta^{(k)}=-\bar{F}\left(y^{(k)}\right)$ with respect to $\eta^{(k)}$;

$y^{(k+1)}:=y^{(k)}+\eta^{(k)} ;$ (new approximation)

evaluate $F\left(y^{(k+1)}\right)$ and compute $\bar{F}\left(y^{(k+1)}\right)$;

until $\left\|\eta^{(k)}\right\|<\varepsilon_{y}$ and $\left\|F\left(y^{(k+1)}\right)\right\|<\varepsilon_{F}$;

$u:=y^{(k+1)} ;$ (curve point found).

Remark 3.8. Note that the repeated computation of tangent vector and inverse involves some numerical linear algebra. These computations can be made more efficient by using $L U$-decompositions: Consider a decomposition of the form

$$
P A^{*}=L\left(\begin{array}{c}
U \\
0^{*}
\end{array}\right)
$$

where $L$ is a lower triangular $(n+1) \times(N+1)$ matrix, $U$ is an upper triangular matrix and $P$ a $(N+1) \times(N+1)$ permutation matrix. For such decompositions in Section 4.5 in Allgower and Georg (1990) are deduced formulae to effectively compute the tangent vector and the Moore-Penrose inverse.

Now we are almost ready to give an algorithm to numerically trace a curve. We will use this algorithm to approximate existence boundaries for equilibria as well as stability boundaries.

To restrict the costly recalculation of the Jacobian, we compute the Jacobian via numerical differentiation merely in the initial guess and then once per approximated curve point, namely in the final approximation; see Fig. 2. In particular, we use the same Jacobian for the predicted point $u+\sigma t$ as for the previous curve point $u$. Note that in general Broyden updates do not converge to the Jacobian, such that for the computation of the tangent in a curve point, we should approximate the Jacobian via numerical differentiation. We include pseudo-code to compute updates of the population birth rate along the curves. This code should be used to compute the value of the birth rate along the stability boundaries or along equilibrium curves in equilibrium parameter space; see Section 2.2.2. When computing existence boundaries the according lines should of course be deleted, as along the existence boundary the population birth rate is zero by definition. 
Algorithm 3.9 (Tracing of curve including updates of population birth rate).

\section{input}

begin

$F: \Omega \longrightarrow \mathbb{R}^{N}, \Omega \subset \mathbb{R}^{N+1} ;$ (map defining curve)

$y^{(0)} \in \mathbb{R}^{N+1} ;$ (initial guess)

evaluation algorithm for $F$;

$\varepsilon_{y}, \varepsilon_{F}>0 ;$ (numerical accuracy constants)

$\delta_{1}, \delta_{2} ;$ (accuracy constants for numerical differentiation)

$\sigma>0$; (initial stepsize)

end

$t:=(1,0, \ldots, 0) ;($ orthonormal vector defining hyperplane)

compute $A \approx F^{\prime}\left(y^{(0)}\right)$ using numerical differentiation and the evaluation algorithm;

$v^{(0)}:=y^{(0)}, k:=0 ;($ start of the Newton iteration $)$;

\section{repeat}

with Algorithm 3.7 approximate and store curve point $u^{(k)}$ with initial guess $v^{(k)}$;

compute $\bar{b}^{k}:=\bar{b}_{\varepsilon}\left(u^{(k)}\right)$ with Algorithm 3.3 and store it; (population birth rate)

approximate $A \approx F^{\prime}\left(u^{(k)}\right)$ using numerical differentiation with accuracy

$\delta$ and the evaluation algorithm;

compute $t(A)$ as in Definition 3.6; (tangent vector)

set $t:=t(A)$;

choose a stepsize $\sigma$;

$k:=k+1$;

set $v^{(k)}:=u^{(k-1)}+\sigma t ;($ prediction)

until traversing is stopped

$U:=\left\{u^{(k)}: k=0,1, \ldots\right\} ;($ set of points tracing curve)

$B:=\left\{\bar{b}^{(k)}: k=0,1, \ldots\right\} ;($ updates of population birth rate).

\subsection{Tracing of existence boundaries}

To trace existence boundaries, we should in Algorithm 3.9 set $N:=2$ and $F:=G_{\varepsilon}$, where $G_{\varepsilon}$ is defined as in (23), choose an initial guess $y^{(0)}:=\left(\alpha_{1}, \alpha_{2}, \bar{S}\right)$ and use Algorithm 3.2 to evaluate $G_{\varepsilon}$. Moreover, as remarked before, we should delete the lines where the population birth rate is computed. We then obtain a set $U:=\left\{\left(\alpha_{1}^{(k)}, \alpha_{2}^{(k)}, \bar{S}^{(k)}\right): k=0,1, \ldots\right\}$, 
such that $\left\{\left(\alpha_{1}^{(k)}, \alpha_{2}^{(k)}\right): k=0,1, \ldots\right\}$ is a tracing of the existence boundary and $\bar{S}^{(k)}$ a tracing of the value of the equilibrium along the existence boundary.

For the implementation for the Daphnia model, we continue the curve defined by (17). In Algorithm 3.9, we should then set $N:=1, F:=G_{\varepsilon}$, where now $G_{\varepsilon}$ is as defined in (25), choose an initial guess $y^{(0)}:=\left(\alpha_{1}, \bar{S}\right)$ and use Algorithm 3.2 to evaluate $G_{\varepsilon}$. We then obtain a set $U:=\left\{\left(\alpha_{1}^{(k)}, \bar{S}^{(k)}\right): k:=0,1, \ldots\right\}$, which gives a tracing of the equilibrium as one parameter changes and a set $B$ of updates of the population birth rate for these values. We then can use $U$ to define the existence boundary for equilibria as $\left\{\left(\alpha_{1}^{(k)}, \alpha_{2}^{(k)}\right)\right.$ : $\left.\alpha_{2}^{(k)}:=\bar{S}^{(k)}, k=0,1, \ldots\right\}$; see Section 2.2.2.

\subsection{Integration for the computation of stability boundaries}

We here show the integration to evaluate the map which defines the stability boundaries and which was defined in Section 2.3.5. The algorithm on its own can be used to test how close to the curve an initial guess is.

Algorithm 3.10 (Integration to evaluate $F_{\varepsilon}$ ).

\section{input}

\section{begin}

$\varepsilon>0 ;$ (survival tolerance)

$\bar{S}, \alpha_{1}, \alpha_{2}, \omega ;$ (given values)

\section{end}

$a_{0}:=0, x_{0}:=x_{b}, f_{0}:=1, k_{r}^{0}:=0, k_{i}^{0}:=0, l_{r}^{0}:=0, l_{i}^{0}:=0, \psi_{3, r}^{0}:=0, \psi_{3, i}^{0}:=0$, $\psi_{J, 4, r}^{0}:=0, \psi_{J, 4, i}^{0}:=0$

integrate in parallel (18)-(19), (41)-(42), and (45)-(46) until

$X(a, \bar{S})=x_{A}$;

set $\bar{\tau}:=a$;

store $\bar{\tau}, \mathcal{F}(\bar{\tau}), K_{r}(\bar{\tau}), K_{i}(\bar{\tau}), L_{r}(\bar{\tau}), L_{i}(\bar{\tau}), \psi_{r}^{3}(\bar{\tau}), \psi_{i}^{3}(\bar{\tau}), \psi_{J, r}^{4}(\bar{\tau})$ and $\psi_{J, i}^{4}(\bar{\tau})$;

$a_{0}:=\bar{\tau}, x_{0}:=x_{A}, f_{0}:=\mathcal{F}(\bar{\tau}), r_{0}:=0, k_{r}^{0}:=K_{r}(\bar{\tau}), k_{i}^{0}:=K_{i}(\bar{\tau}), l_{r}^{0}:=L_{r}(\bar{\tau})$, $l_{i}^{0}:=L_{i}(\bar{\tau}), \psi_{1, r}^{0}:=0, \psi_{1, i}^{0}:=0$,

$$
\begin{aligned}
& \psi_{2, r}^{0}:=\frac{\mathcal{F}(\bar{\tau}) \beta^{+}}{g^{-}} K_{r}^{e}(\bar{\tau}, \bar{\tau}), \quad \psi_{2, i}^{0}:=\frac{\mathcal{F}(\bar{\tau}) \beta^{+}}{g^{-}} K_{i}^{e}(\bar{\tau}, \bar{\tau}), \\
& \psi_{3, r}^{0}:=\psi_{r}^{3}(\bar{\tau}), \quad \psi_{3, i}^{0}:=\psi_{i}^{3}(\bar{\tau}), \\
& \psi_{4, r}^{0}:=\psi_{J, r}^{4}(\bar{\tau})-\frac{\mathcal{F}(\bar{\tau})}{g^{-}}\left(\gamma^{+}-\gamma^{-}\right) K_{r}^{e}(\bar{\tau}, \bar{\tau}), \\
& \psi_{4, i}^{0}:=\psi_{J, i}^{4}(\bar{\tau})-\frac{\mathcal{F}(\bar{\tau})}{g^{-}}\left(\gamma^{+}-\gamma^{-}\right) K_{i}^{e}(\bar{\tau}, \bar{\tau}) ;
\end{aligned}
$$


integrate in parallel (18)-(20), (41)-(42), (43)-(45), and (47) until $\mathcal{F}(a)=\varepsilon$;

$a_{\varepsilon}:=a ;$

store $r\left(a_{\varepsilon}, \bar{S}\right), \psi_{r}^{j}\left(a_{\varepsilon}\right)$ and $\psi_{i}^{j}\left(a_{\varepsilon}\right), j=\{1, \ldots, 4\}$;

compute and store $G_{\varepsilon}\left(\alpha_{1}, \alpha_{2}, \bar{S}\right):=r\left(a_{\varepsilon}, \bar{S}\right)-1$;

compute $f(\bar{S})$ and $f^{\prime}(\bar{S})$ and $H_{\varepsilon}\left(\alpha_{1}, \alpha_{2}, \bar{S}, \omega\right)$ via (48);

compute $F_{\varepsilon}\left(\alpha_{1}, \alpha_{2}, \bar{S}, \omega\right)$ as in (49).

\subsection{Tracing of the stability boundary}

To trace stability boundaries in two parameter space, we set in Algorithm $3.9 N:=3$, $F:=F_{\varepsilon}$ with $F_{\varepsilon}$ as in (49), fix an initial guess $y^{(0)}:=\left(\alpha_{1}, \alpha_{2}, \bar{S}, \omega\right)$ and pass on Algorithm 3.10 to evaluate $F_{\varepsilon}$. We then obtain sets $U:=\left\{\left(\alpha_{1}^{(k)}, \alpha_{2}^{(k)}, \bar{S}^{(k)}, \omega^{(k)}\right): k=0,1, \ldots\right\}$ and $B$ that give a tracing of the stability boundary in the two parameter plane as well as updates of the equilibrium including population birth rate along he curve.

Remark 3.11. Note that in (49) the first component is independent of $\omega$, which is a general result for stability boundaries. Moreover, in the case of Daphnia models, the first component in (49) is, as mentioned, additionally independent of one of the two parameters. This raises the question, whether this structure can be exploited to make computations more efficient. As one example, in every evaluation of the function $H_{\varepsilon}$ in (49) a Newton iteration could be implemented to satisfy $G_{\varepsilon}\left(\alpha_{1}, \alpha_{2}, \bar{S}\right)=0$, and thus compute $\bar{S}$ for given $\alpha_{1}, \alpha_{2}$, which effectively wraps up a Newton iteration to satisfy the equilibrium condition into a Newton iteration for locating the solution to the stability condition. Since algorithms for a single Newton iteration are easier to implement and since the resulting code is easier to read and for our present low-dimensional problems sufficient, we leave this question for future research.

\section{Implementations for Daphnia models}

In this section, we specify the ingredients for different parametrizations for models of a length structured Daphnia population consuming an unstructured algae population from de Roos et al. (1990) and de Roos (1997) and show the results of the computations of existence and stability boundaries in the form of graphs. For more precise biological interpretation of the graphs, we refer to de Roos et al. (1990) and de Roos (1997).

\subsection{Model ingredients}

We denote the length of a Daphnia individual by $x$ and by $S$ the concentration of algae. For meanings and values of parameters, we refer to Table 1 . In the absence of consumption, the dynamics of an algae population $S$ are described by a rate $f(S)=a_{1}\left(S_{\max }-S\right)$ for chemostat models or $f(S)=a_{2} S\left(1-\frac{S}{S_{\max }}\right)$ for logistic models. We here disregard the 
Table 1 Parameter specification as in de Roos et al. (1990)

\begin{tabular}{|c|c|c|c|}
\hline Description & Symbol & Value & Unit \\
\hline Length at birth & $x_{b}$ & 0.8 & $\mathrm{~mm}$ \\
\hline Length at maturation & $x_{A}$ & 2.5 & $\mathrm{~mm}$ \\
\hline $\begin{array}{l}\text { Maximum attainable length } \\
\text { under infinite food availability }\end{array}$ & $x_{m}$ & 6.0 & $\mathrm{~mm}$ \\
\hline Time constant of growth & $\gamma_{g}$ & 0.15 & $\mathrm{~d}^{-1}, \mathrm{~d}=$ day \\
\hline $\begin{array}{l}\text { Shape parameter of the } \\
\text { functional response }\end{array}$ & $\xi$ & $7.0 \times 10^{-6}$ & $\mathrm{ml} \cdot$ cell $^{-1}$ \\
\hline $\begin{array}{l}\text { Maximum feeding rate } \\
\text { per unit surface area }\end{array}$ & $v_{S}$ & $1.8 \times 10^{6}$ & cell $\cdot \mathrm{mm}^{-2} \cdot \mathrm{d}^{-1}$ \\
\hline $\begin{array}{l}\text { Fraction of ingested energy } \\
\text { channeled to growth and maintenance }\end{array}$ & $\kappa$ & 0.3 & - \\
\hline $\begin{array}{l}\text { Maximum reproduction rate } \\
\text { per unit surface area }\end{array}$ & $r_{m}$ & 0.1 & $\mathrm{~mm}^{-2} \cdot \mathrm{d}^{-1}$ \\
\hline Random death rate of Daphnia & $\mu$ & variable & $\mathrm{d}^{-1}$ \\
\hline $\begin{array}{l}\text { Maximum concentration of algae } \\
\text { in the absence of Daphnia }\end{array}$ & $S_{\max }$ & variable & cell $\cdot \mathrm{ml}^{-1}$ \\
\hline $\begin{array}{l}\text { Flow-through rate in case of } \\
\text { chemostat algal dynamics }\end{array}$ & $a_{1}$ & 0.5 & $d^{-1}$ \\
\hline $\begin{array}{l}\text { Flow-through rate in case of } \\
\text { logistic algal dynamics }\end{array}$ & $a_{2}$ & 0.5 & $\mathrm{~d}^{-1}$ \\
\hline
\end{tabular}

equilibria $(\bar{b}, \bar{S})=\left(0, S_{\max }\right)$ and $(\bar{b}, \bar{S})=(0,0)$ and concentrate on interior equilibria. The equilibrium growth rate is given as

$$
g(x, \bar{S})=\gamma_{g}\left(x_{m} f_{r}(\bar{S})-x\right),
$$

where here and in the following we denote the (Holling type II) functional response as

$$
f_{r}(\bar{S}):=\frac{\xi \bar{S}}{1+\xi \bar{S}} .
$$

The equilibrium birth rate and consumption rate become

$$
\begin{aligned}
& \beta(x, \bar{S})= \begin{cases}0, & x_{b} \leq x \leq x_{A}, \\
r_{m} f_{r}(\bar{S}) x^{2}, & x_{A} \leq x,\end{cases} \\
& \gamma(x, \bar{S})=v_{S} f_{r}(\bar{S}) x^{2} .
\end{aligned}
$$

Finally, we assume that the mortality rate is a constant, which we also denote by $\mu$, i.e., that $\mu(x, \bar{S}) \equiv \mu$. In de Roos et al. (1990), it is motivated that from a biological point of view a good choice for the two free parameters is the background mortality for Daphnia $\mu$ and the carrying capacity for Algae $S_{\max }$. For the computation of existence boundaries, we use the special parameter dependence for Daphnia in the way that we use the second of the two methods described in Section 3.4, i.e., we define $F:=G_{\varepsilon}$ and $G_{\varepsilon}$ as 
Table 2 Numerical integration with Algorithms 3.2 and 3.3

\begin{tabular}{llll}
\hline $\bar{S}$ & $\mu$ & $\bar{b}_{\varepsilon}(\bar{S}, \mu)$ & $G_{\varepsilon}(\bar{S}, \mu)$ \\
\hline $5.000000 \mathrm{E}+05$ & 0.300000 & $5.156976 \mathrm{E}-03$ & -0.133576 \\
\hline
\end{tabular}

in (25). Next, we recall that we locate the first curve point by fixing $\alpha_{1}$ and then applying a Newton algorithm to find the other components of the curve point. In this regard for the Daphnia models, a good choice is to set $\alpha_{1}:=\mu$ for the computations of existence boundaries and $\alpha_{1}:=S_{\max }$ and $\alpha_{2}:=\mu$ for stability boundaries. As accuracy constants for numerical differentiation, we use $\delta_{1}:=1.0 \mathrm{E}-4, \delta_{2}:=1.0 \mathrm{E}-7$. Finally, in the computations where integration is stopped by decreased survival, we define $\varepsilon:=10^{-9}$ as the survival probability at which integration stops. If we stop integration when $a$ reaches $A_{\max }$, we set $A_{\text {max }}=70$ years.

\subsection{Existence boundaries}

In the following, we show the results of the implementation of Algorithms 3.2, 3.3, 3.7, and 3.9.

\subsubsection{Numerical integration for existence boundaries}

We show a numerical example for the computation of $G_{\varepsilon}(\bar{S}, \mu)$ and $\bar{b}_{\varepsilon}(\bar{S}, \mu)$ for given $\bar{S}, \mu$ with Algorithms 3.2 and 3.3 in Table 2. The isolated Algorithm 3.2 can be used to find out how close to the curve a guess is, and thus also to find an initial guess which leads to convergence. The numerical example shown is such a guess. We stop integration when $a$ reaches $A_{\max }$.

\subsubsection{Tracing of existence boundaries}

We have traced existence boundaries for Daphnia models with Algorithms 3.2, 3.7, and 3.9. For Daphnia magna as parametrized in Table 1, this is the dotted line in Figs. 3-4 and 6-9.

In Figs. 11-13, we have plotted the data for the model of Daphnia pulex feeding on Chlamydomonas rheinhardii in (de Roos, 1997). The model has the same specification of vital rates in terms of parameters as the Daphnia magna model, but the parameters have different values. The values used are now $x_{b}=0.6, x_{A}=1.4, x_{m}=3.5, F_{h}=\frac{1}{\xi}=0.164$, $\gamma_{g}=0.11, r_{m}=1.0, v_{S}:=0.007$, and $a_{2}:=0.5$. We use the same values for stopping the integration as for the Daphnia magna model.

\subsection{Stability boundaries}

To specify the ODE for the computation of stability boundaries, it remains to compute the partial derivatives of the vital rates in a point $(X(a, \bar{S}), \bar{S})$, as well as their one-sided limits in $x_{A}$ for given $\bar{S}$. These partial derivatives are in the notation of Section 2.3.3

$$
\begin{aligned}
& g_{1}(a)=-\gamma_{g}, \quad g_{2}(a)=\gamma_{g} x_{m} f_{r}^{\prime}(\bar{S}), \quad f_{r}^{\prime}(\bar{S})=\frac{\xi}{(1+\xi \bar{S})^{2}}, \\
& \mu_{1}(a)=0, \quad \mu_{2}(a)=0,
\end{aligned}
$$


Table 3 Numerical integration with Algorithm 3.10. $H_{\varepsilon, 1}$ and $H_{\varepsilon, 2}$ denote the two components of $H_{\varepsilon}$

\begin{tabular}{llll}
\hline $\begin{array}{l}\text { input } \\
\bar{S}\end{array}$ & $\mu$ & $S_{\max }$ & $\omega$ \\
\hline $1.032074 \mathrm{E}-01$ & $4.925993 \mathrm{E}-02$ & $2.000000 \mathrm{E}+00$ & $3.455190 \mathrm{E}-01$ \\
\hline output & $H_{\varepsilon, 1}$ & $H_{\varepsilon, 2}$ & \\
$G_{\varepsilon}$ & $4.042150 \mathrm{E}-02$ & $-7.720165 \mathrm{E}-01$ & \\
\hline $1.714976 \mathrm{E}-01$ & & & \\
\hline
\end{tabular}

$$
\begin{aligned}
& \beta_{1}(a)= \begin{cases}0, & x_{b} \leq X(a, \bar{S})<x_{A}, \\
2 r_{m} f_{r}(\bar{S}) X(a, \bar{S}), & x_{A} \leq X(a, \bar{S}),\end{cases} \\
& \beta_{2}(a)= \begin{cases}0, & x_{b} \leq X(a, \bar{S})<x_{A}, \\
r_{m} f_{r}^{\prime}(\bar{S}) X^{2}(a, \bar{S}), & x_{A} \leq X(a, \bar{S}),\end{cases} \\
& \gamma_{1}(a)=2 v_{S} f_{r}(\bar{S}) X(a, \bar{S}), \quad \gamma_{2}(a)=v_{S} f_{r}^{\prime}(\bar{S}) X^{2}(a, \bar{S}) .
\end{aligned}
$$

The one-sided limits are

$$
\begin{array}{ll}
g_{-}=g_{+}=\gamma_{g}\left(x_{m} f_{r}(\bar{S})-x_{A}\right), & \mu_{-}=\mu_{+}=\mu, \\
\beta_{-}=0, \quad \beta_{+}=r_{m} f_{r}(\bar{S}) x_{A}{ }^{2}, & \gamma_{-}=\gamma_{+}=v_{S} f_{r}(\bar{S}) x_{A}{ }^{2} .
\end{array}
$$

\subsubsection{Numerical integration for stability boundaries}

In Table 3, we show an example of the computed values of $F_{\varepsilon}\left(\bar{S}, \mu, S_{\max }, \omega\right)$ for given $\bar{S}$, $\mu, S_{\max }$, and $\omega$ with Algorithm 3.10. Like in the one-parameter problem, we can use the isolated algorithm for numerical integration to find an initial guess that leads to convergence. We show such a guess in Table 3, where we stopped integration at $a=A_{\max }$.

\subsubsection{Stability boundaries}

We then trace stability boundaries for Daphnia magna with Algorithm 3.9, which calls Algorithm 3.10 for numerical integration, Algorithm 3.7 to locate the first point on the curve and for correction and Algorithm 3.3 for updates of the population birth rate. The resulting curves are shown in Figs. 3-10. In Figs. 4, 7, and 9, we have used the reaching of $A_{\max }$ as stopping mechanism to have the same conditions as in de Roos et al. (1990). The figures correspond with Fig. 2 in de Roos et al. (1990). In Figs. 11 and 13, we have computed the stability boundaries for the Daphnia pulex model in de Roos (1997). Figure 13 coincides with Fig. 7 in this reference. In Fig. 5, resp. 10 and 12, we depict the same graph as in Fig. 4, resp. 9 and 11, on larger scales to show details, such as windings of the curves.

In the existence region near to the existence boundary, the number of roots in the righthalf plane is zero. We call the computed curve suggestively a stability boundary, since we believe, on the basis of numerical solutions of initial value problems (de Roos et al., 1990), that this number stays zero up to the curve. Upon crossing the computed curve in a regular 


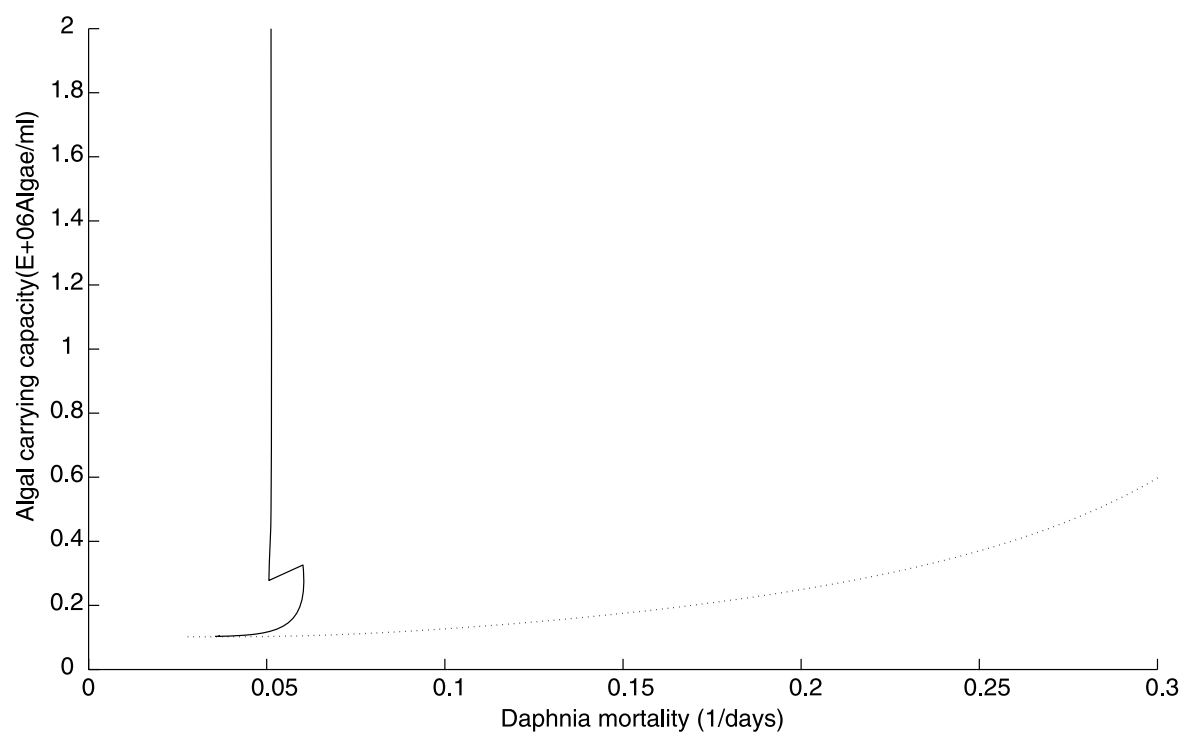

Fig. 3 Chemostat algal dynamics $\left(a_{1}=0.5\right)$, integration stopped when survival drops below the threshold $10^{-9}$.

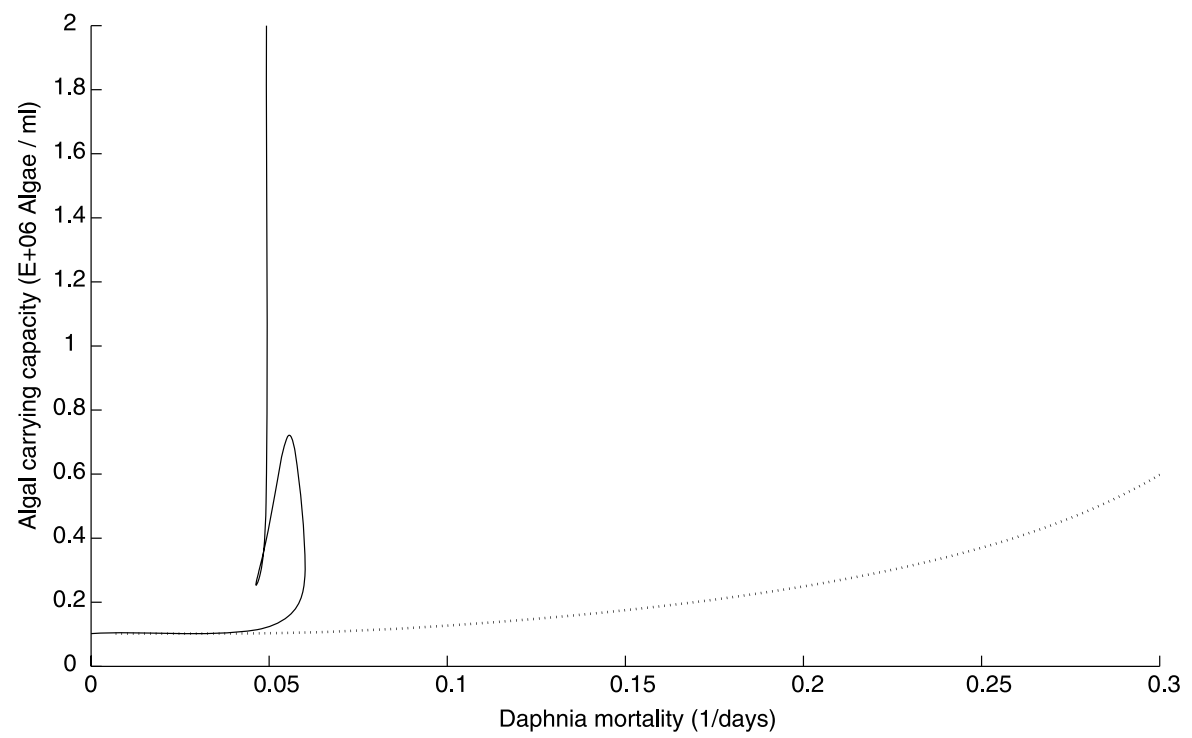

Fig. 4 Chemostat algal dynamics $\left(a_{1}=0.5\right)$, integration stopped at $a=A_{\max }=70$ years.

point the number of roots in the right-half plane changes from zero to two. If the curve intersects itself, like in Fig. 5, the number of roots in the right-half plane equals four in the domain bounded by the closed loop. We made no effort to compute other solution curves 


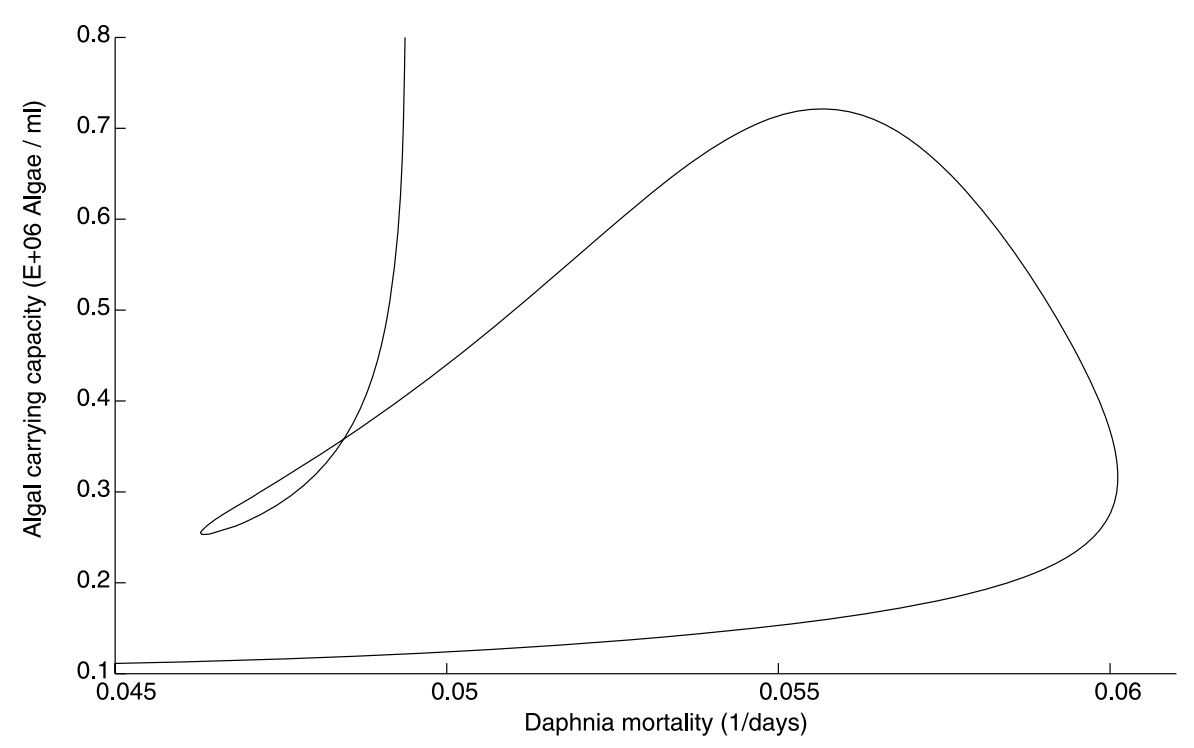

Fig. 5 Chemostat algal dynamics $\left(a_{1}=0.5\right)$, integration stopped at $a=A_{\max }=70$ years.

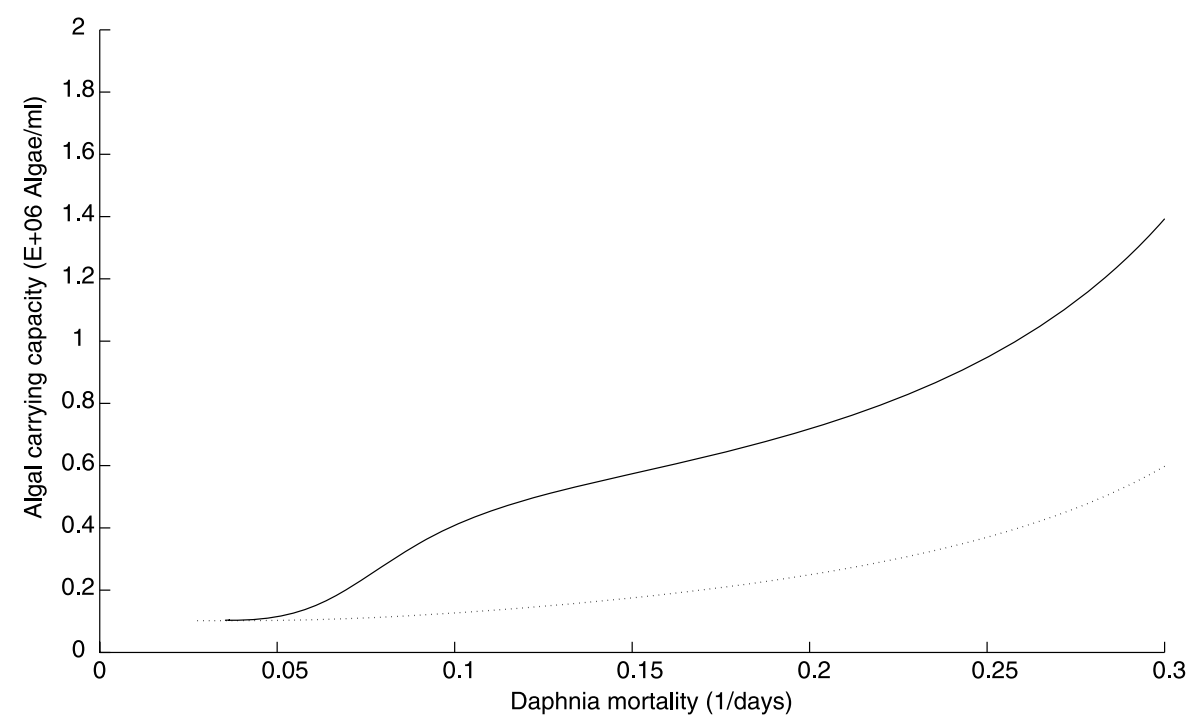

Fig. 6 Logistic algal dynamics $\left(a_{2}=0.5\right)$, integration stopped when survival drops below the threshold $10^{-9}$.

of (32) at which additional pairs of roots move into the right-half plane. Moreover, we believe, again on the basis of numerical solutions of initial value problems (de Roos et al., 1990) that stability of the steady state cannot be regained in the region to the left of the 


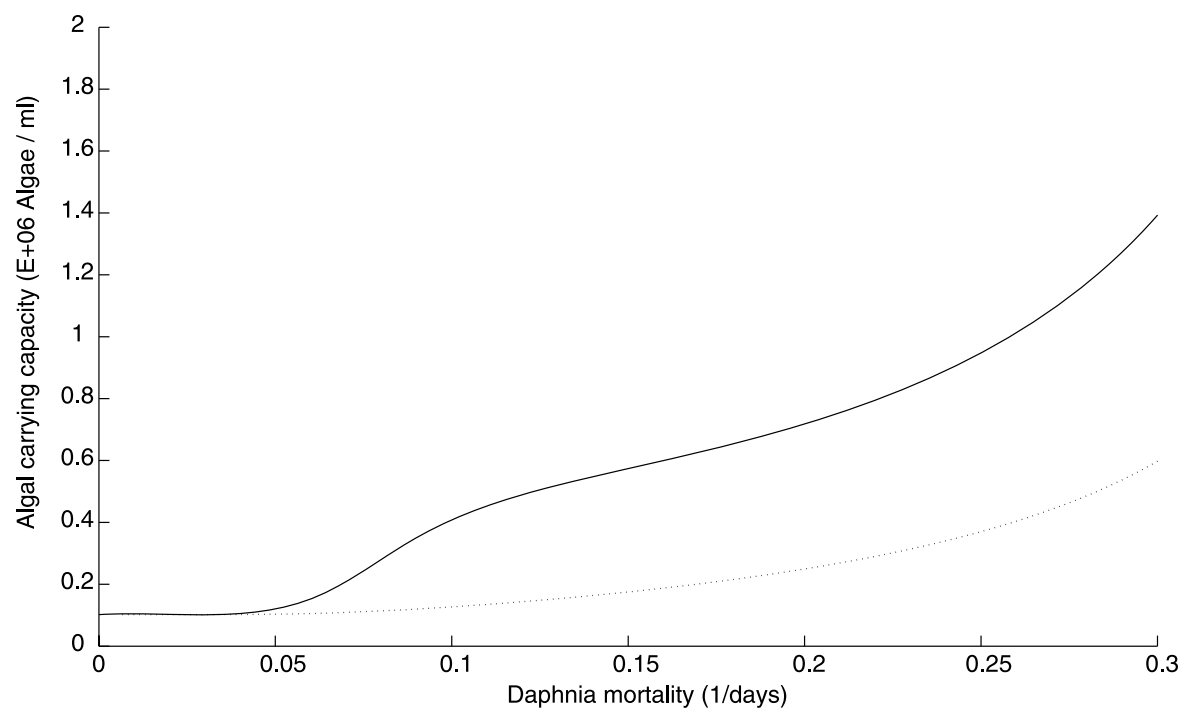

Fig. 7 Logistic algal dynamics $\left(a_{2}=0.5\right)$, integration stopped at $a=A_{\max }=70$ years.

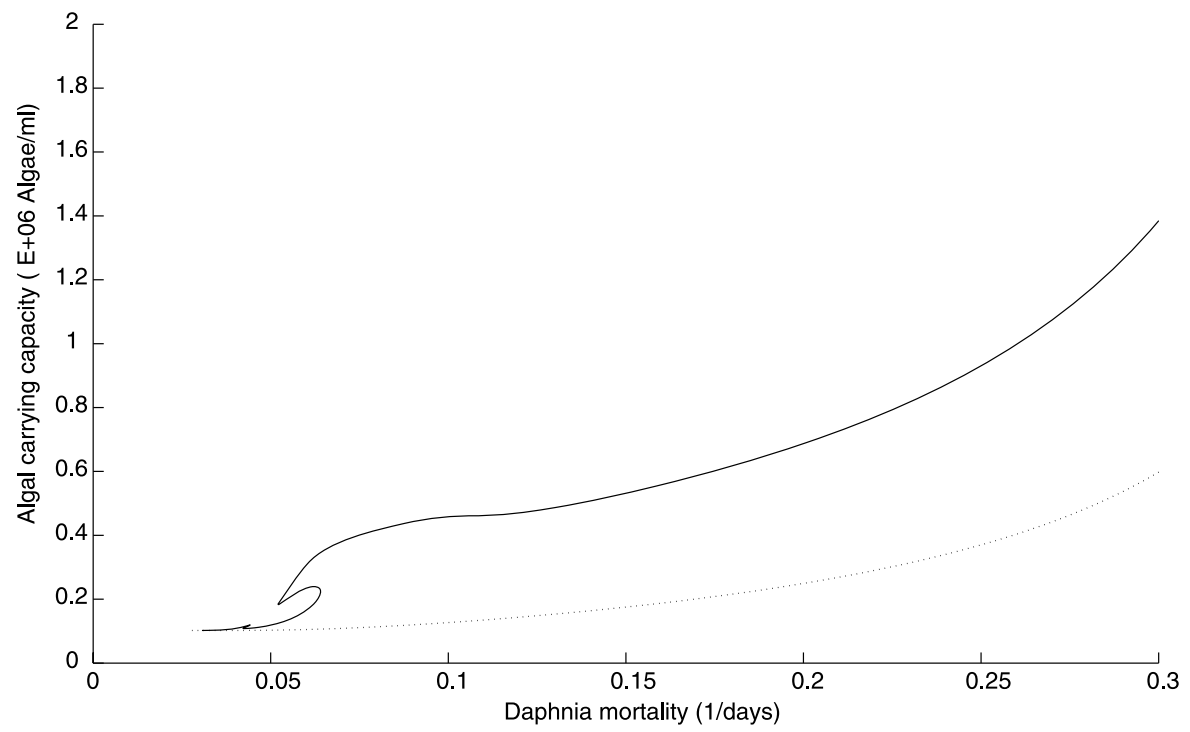

Fig. 8 Logistic algal dynamics $\left(a_{2}=2.0\right)$, integration stopped when survival drops below the threshold $10^{-9}$.

computed stability boundary. In summary, on the basis of numerical evidence we believe that the computed curves are the stability boundary of the steady state in the parameter plane that we consider. 


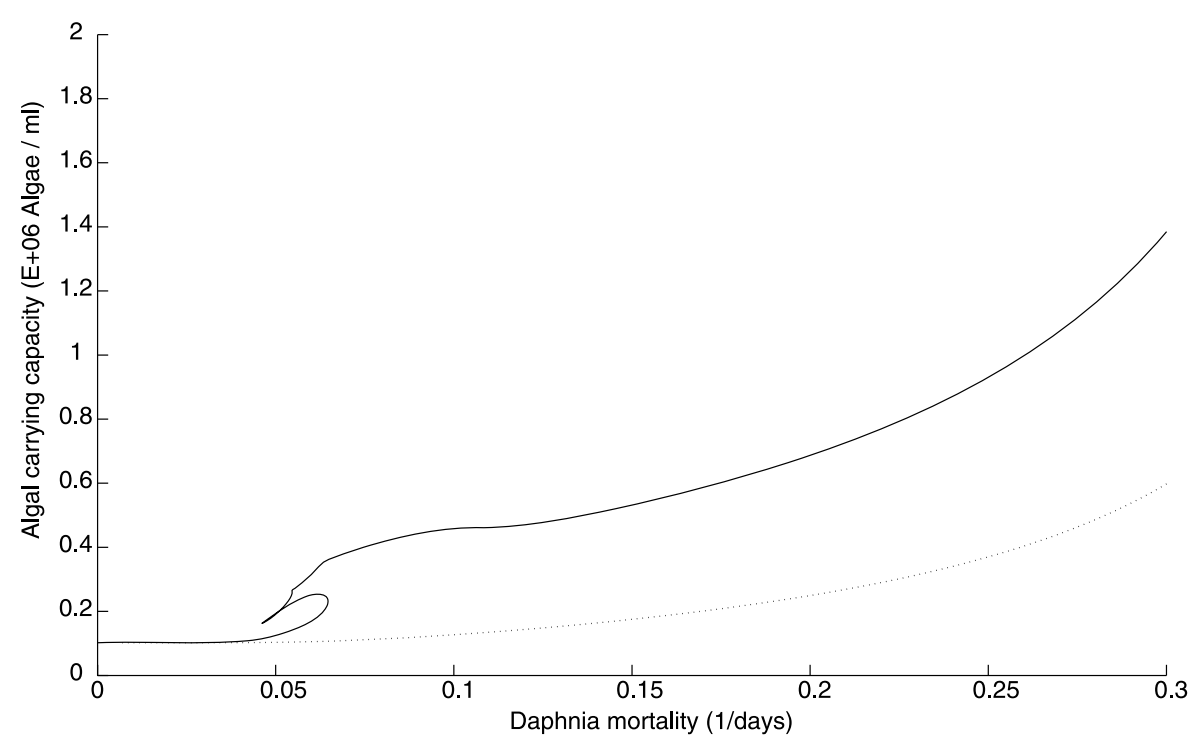

Fig. 9 Logistic algal dynamics $\left(a_{2}=2.0\right)$, integration stopped at $a=A_{\max }=70$ years.

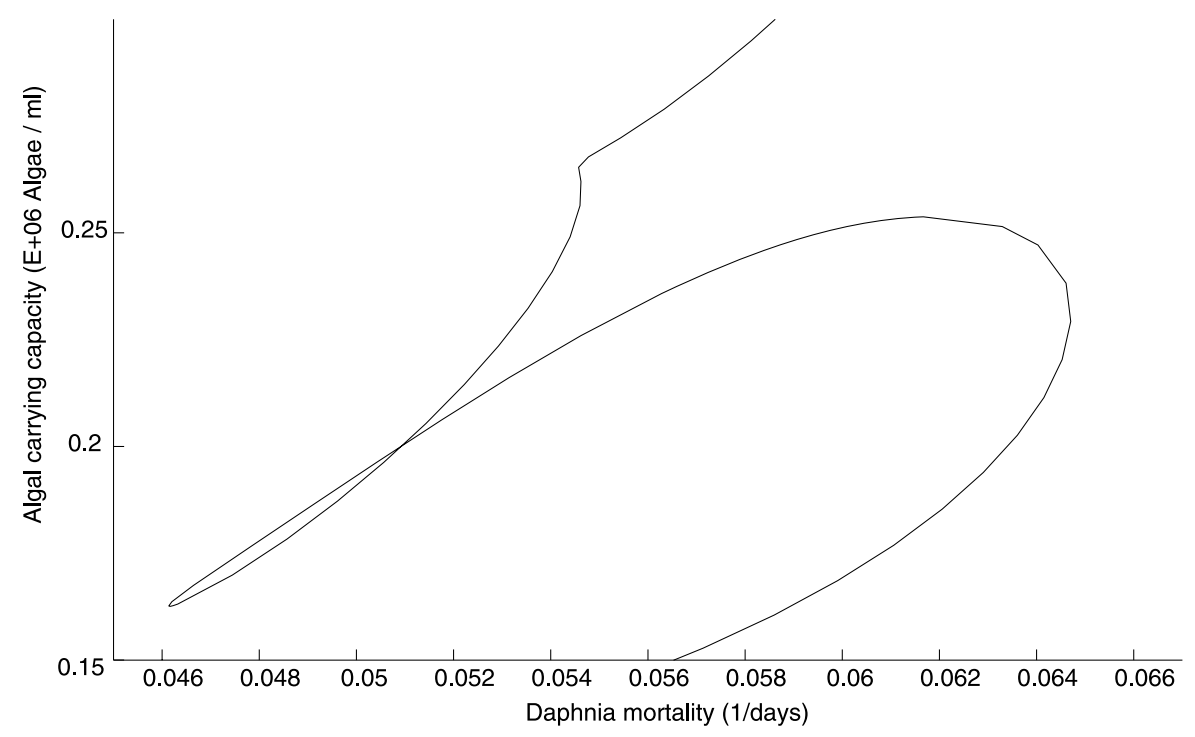

Fig. 10 Logistic algal dynamics $\left(a_{2}=2.0\right)$, integration stopped at $a=A_{\max }=70$ years.

\section{Concluding remarks}

Building on earlier work, we have developed methods to trace equilibrium curves and stability boundaries for a class of structured consumer resource models. We have im- 


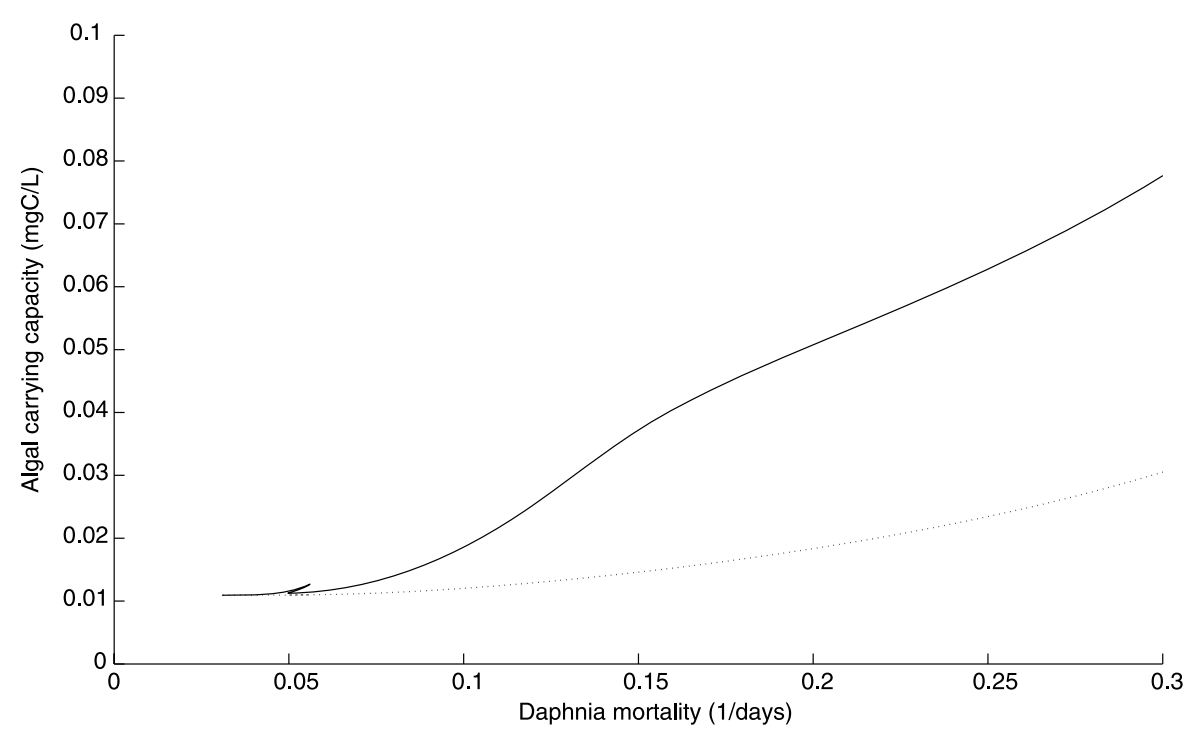

Fig. 11 Daphnia pulex, integration stopped when survival drops below the threshold $10^{-9}$.

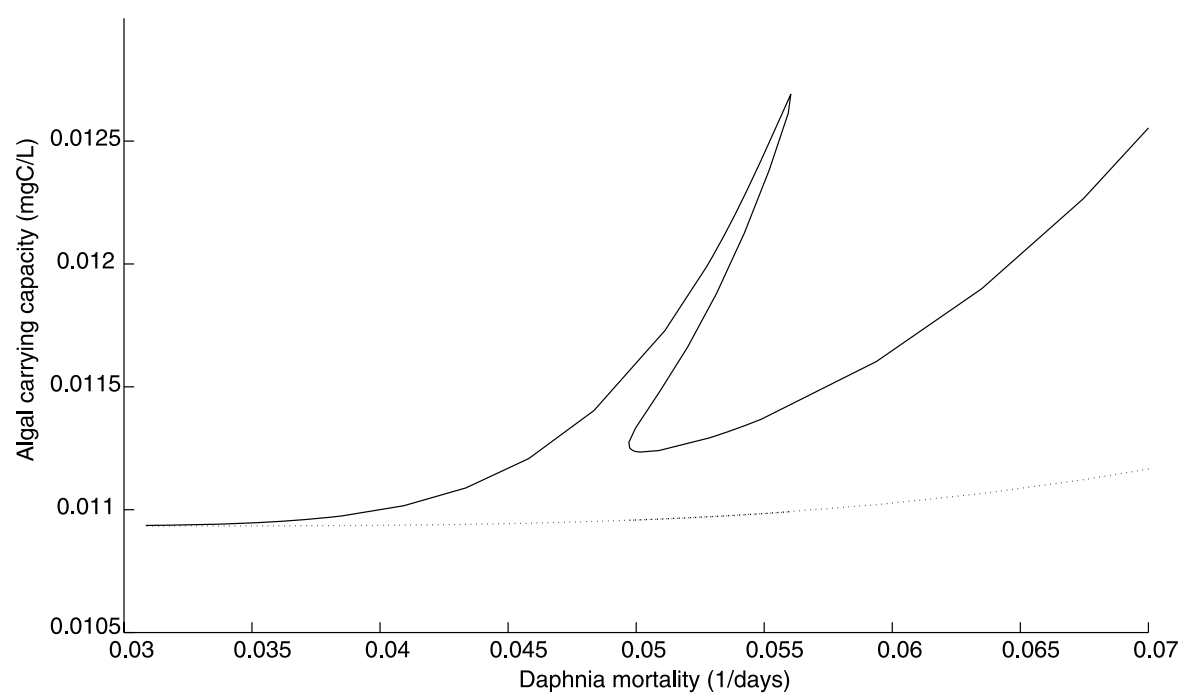

Fig. 12 Daphnia pulex, integration stopped when survival drops below the threshold $10^{-9}$.

plemented the resulting algorithms in a C-code for existing models of Daphnia magna and Daphnia pulex consuming algae. In the class of models we analyzed, we assumed a fixed state at which individuals are born. Moreover, we incorporated the competition of a structured consumer population for an unstructured resource. 


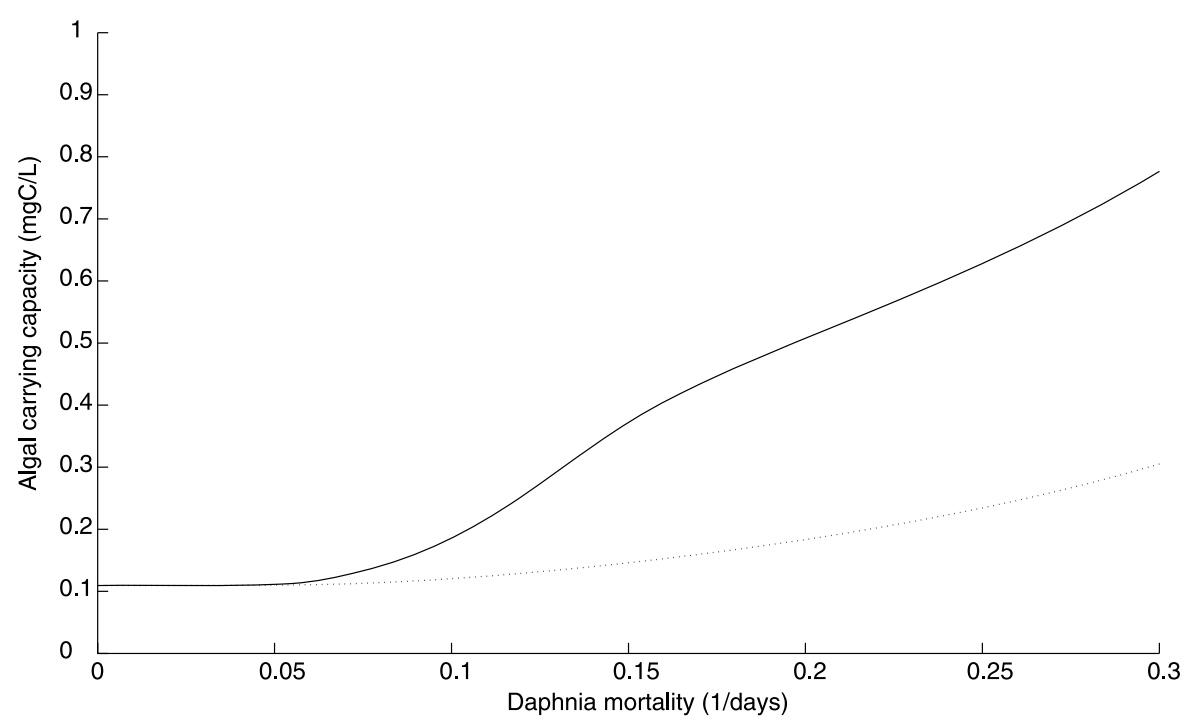

Fig. 13 Daphnia pulex, integration stopped at $a=A_{\max }=70$ years.

Our next objective is to perform a similar analysis for different biological phenomena. Depending on the nature of these, we can either use the theory established here or try to generalize this theory. Possible relevant generalizations may include

- allowing for multiple states at birth

- incorporating further interactions, e.g. the competition for several unstructured resources (which leads to multiple component delay differential equations) and/or ingestion and predation among structured populations (which leads to multiple component renewal equations)

- allowing for infinitely many birth states or interactions (or both).

We would therefore like to encourage our readers to approach us with biological models or phenomena that could be analyzed with the approach developed in this paper or with the approach extended by including one or more of the above points.

With several biological examples at hand, it will make sense to transform our code with, e.g., Matlab, to a more user-friendly tool that has computations such as the deduction of partial derivatives or the realification of ODE automatized.

\section{Acknowledgements}

Part of PhG's research was carried out at the Department of Mathematics of the University of Warwick supported by the Sixth Framework Programme Uninet (Unifying Networks for Science and Society). PhG would like to thank Luca Sbano and Martin Eigel for valuable discussions during this time. The research of OD and PhG has been supported by and has benefited from the Research Programme Mathematical Biology that was organized by the CRM in Barcelona in 2009. We thank Sebastiaan Janssens for helpful comments on the manuscript. 
Open Access This article is distributed under the terms of the Creative Commons Attribution Noncommercial License which permits any noncommercial use, distribution, and reproduction in any medium, provided the original author(s) and source are credited.

\section{Appendix A: Derivation of the characteristic equation}

We show that formal linearization of (6)-(7) leads to the characteristic equation (26)-(27) with the functions $\Psi^{i}$ as defined in (34)-(36). For differentiability proofs, we refer to de Roos et al. (2009). We denote by $y$ and $z$ small perturbations of $\bar{b}$ and $\bar{S}$ respectively. Linearization of (6)-(7) at $(\bar{b}, \bar{S})$ in the notation of Section 2.3.3 yields

$$
\begin{aligned}
y(t)= & \int_{\bar{\tau}}^{h} \beta(a) \mathcal{F}(a) y(t-a) d a-\bar{b} \beta^{+} \mathcal{F}(\bar{\tau}) D a_{A}(\bar{S}) z_{t} \\
& +z(t) \bar{b} \int_{\bar{\tau}}^{h} \beta_{2}(a) \mathcal{F}(a) d a+\bar{b} \int_{\bar{\tau}}^{h} \mathcal{F}(a) \beta_{1}(a) D_{2} X(a, \bar{S}) z_{t} d a \\
& +\bar{b} \int_{\bar{\tau}}^{h} \beta(a) D_{2} \mathcal{F}(a, \bar{S}) z_{t} d a \\
z^{\prime}(t)= & f^{\prime}(\bar{S}) z(t)-\int_{0}^{h} \gamma(a) \mathcal{F}(a) y(t-a) d a-\bar{b}\left(\gamma^{-}-\gamma^{+}\right) \mathcal{F}(\bar{\tau}) D a_{A}(\bar{S}) z_{t} \\
& -z(t) \bar{b} \int_{0}^{h} \gamma_{2}(a) \mathcal{F}(a) d a-\bar{b} \int_{0}^{h} \mathcal{F}(a) \gamma_{1}(a) D_{2} X(a, \bar{S}) z_{t} d a \\
& -\bar{b} \int_{0}^{h} \gamma(a) D_{2} \mathcal{F}(a, \bar{S}) z_{t} d a
\end{aligned}
$$

where the derivatives with respect to an infinite dimensional variable $D a_{A}(\bar{S}), D_{2} X(a, \bar{S})$, and $D_{2} \mathcal{F}(a, \bar{S})$ have to be computed yet. We first compute $D_{2} X(a, \bar{S})$. Linearization of (8) leads to the definition of a function $\eta(\tau)=\eta(\tau ; a, \psi)$ as the solution of

$$
\begin{aligned}
\eta^{\prime}(\tau) & =g_{1}(\tau) \eta(\tau)+g_{2}(\tau) \psi(-a+\tau), \\
\eta(0) & =0 .
\end{aligned}
$$

For later use, we plug in $\psi=z_{t}$ and subsequently $z(\alpha)=e^{\lambda \alpha} \bar{S}$. Then we can equivalently define

$$
\eta(\tau):=\bar{S} e^{\lambda(t-a)} K(\tau, \lambda)
$$

and $K$ as the solution of (37) for $a_{0}=0$ and $k^{0}=0$. The advantage of this transformation, is that the system (37) is due to the independence of $t$ and $a$ in our context easier to integrate than (A.3). Then we can define

$$
D_{3} x(\bar{\tau} ; a, \bar{S}) \psi:=\eta(\tau ; a, \psi)
$$


for $\tau \leq \bar{\tau}$ and $\bar{\tau}$ defined via $x(\bar{\tau} ; a, \bar{S})=x_{A}$, which is independent of $a$. Next, we will show that for $\tau>\bar{\tau}$ the right-hand side of (A.5) gets a correction term due to the discontinuity in $x_{A}$. Let $\tau>\bar{\tau}$. By the implicit function theorem, there exists a unique $\tilde{\tau}(a, \psi)$ for $a>\bar{\tau}$ and $\psi$ in a neighborhood of $\bar{S}$ such that

$$
x(\tilde{\tau}(a, \psi) ; a, \psi)=x_{A} .
$$

As $\bar{\tau}=\tilde{\tau}(a, \bar{S})$, we have that $\tau>\tilde{\tau}(a, \bar{S})$. Then for any $\psi$ there exists a small $\varepsilon$, such that also $\tau>\tilde{\tau}(a, \bar{S}+\varepsilon \psi)$. Then

$$
\begin{aligned}
x( & \tau ; a, \bar{S}+\varepsilon \psi)-x(\tau ; a, \bar{S}) \\
= & x_{A}+\int_{\tilde{\tau}(a, \bar{S}+\varepsilon \psi)}^{\tau} g(x(\sigma ; a, \bar{S}+\varepsilon \psi), \bar{S}+\varepsilon \psi(-a+\sigma)) d \sigma \\
& \quad-x_{A}-\int_{\bar{\tau}}^{\tau} g(x(\sigma ; a, \bar{S}), \bar{S}) d \sigma \\
= & \int_{\tilde{\tau}\left(a, \bar{S}_{+\varepsilon \psi)}\right.}^{\bar{\tau}} g(x(\sigma ; a, \bar{S}), \bar{S}) d \sigma \\
& +\int_{\tilde{\tau}(a, \bar{S}+\varepsilon \psi)}^{\tau}[g(x(\sigma ; a, \bar{S}+\varepsilon \psi), \bar{S}+\varepsilon \psi(-a+\sigma))-g(x(\sigma ; a, \bar{S}), \bar{S})] d \sigma \\
= & -g^{+} D_{2} \tilde{\tau}(a, \bar{S}) \psi \varepsilon-\left[g^{+}-g^{+}\right] D_{2} \tilde{\tau}(a, \bar{S}) \psi \varepsilon \\
& +\varepsilon(\eta(\tau ; a, \psi)-\eta(\bar{\tau} ; a, \psi))+o(\varepsilon),
\end{aligned}
$$

where we used that

$$
\begin{aligned}
& \left.\frac{d}{d \varepsilon} \int_{\bar{\tau}}^{\tau} g(x(\sigma ; a, \bar{S}+\varepsilon \psi), \bar{S}+\varepsilon \psi(-a+\sigma)) d \sigma\right|_{\varepsilon=0} \\
& \quad=\eta(\tau ; a, \psi)-\eta(\bar{\tau} ; a, \psi),
\end{aligned}
$$

which is derived as follows. For $\sigma>\bar{\tau}$, one shows that the map

$$
\left.\sigma \longmapsto \frac{d}{d \varepsilon} x(\sigma ; a, \bar{S}+\varepsilon \psi)\right|_{\varepsilon=0}
$$

solves (A.3), such that by uniqueness

$$
\left.\frac{d}{d \varepsilon} x(\sigma ; a, \bar{S}+\varepsilon \psi)\right|_{\varepsilon=0}=\eta(\sigma) .
$$

The rest follows if one uses that $\eta$ solves (A.3). Then from (A.7), we deduce that

$$
\begin{aligned}
\lim _{\varepsilon \downarrow 0} & \frac{1}{\varepsilon}(x(\tau ; a, \bar{S}+\varepsilon \psi)-x(\tau ; a, \bar{S})) \\
\quad & =-g^{+} D_{2} \tilde{\tau}(a, \bar{S}) \psi+\eta(\tau ; a, \psi)-\eta(\bar{\tau} ; a, \psi) .
\end{aligned}
$$


Since the left-hand side equals $D_{3} x(\tau ; a, \bar{S}) \psi$, we get that

$$
D_{3} x(\tau ; a, \bar{S}) \psi=-g^{+} D_{2} \tilde{\tau}(a, \bar{S}) \psi+\eta(\tau)-\eta(\bar{\tau}) .
$$

It remains to compute $D_{2} \tilde{\tau}(a, \bar{S}) \psi$. If we differentiate (A.6) with respect to $\psi$ evaluated at $\bar{S}$ we get

$$
\left.\frac{\partial}{\partial \tau} x(\tau ; a, \bar{S})\right|_{\tau=\bar{\tau}} D_{2} \tilde{\tau}(a, \bar{S}) \psi+D_{3} x(\bar{\tau} ; a, \bar{S}) \psi=0
$$

from which follows that

$$
D_{2} \tilde{\tau}(a, \bar{S}) \psi=-\frac{\eta(\bar{\tau} ; a, \psi)}{g^{-}} .
$$

If we combine this identity with (A.8) and use the corresponding result for $\tau \leq \bar{\tau}$, we get that

$$
D_{3} x(\tau ; a, \bar{S}) \psi=\eta(\tau)+\eta(\bar{\tau})\left(\frac{g^{+}}{g^{-}}-1\right) H(\tau-\bar{\tau}),
$$

where by $H$ we denote the Heaviside-function. Since in particular

$$
D_{2} X(a, \bar{S}) \psi=D_{3} x(a ; a, \bar{S}) \psi
$$

for the case $\psi=z_{t}$ and $z(\alpha)=\bar{S} e^{\lambda \alpha}$ we can use (A.4) and obtain that

$$
D_{2} X(a, \bar{S}) z_{t}=\bar{S} e^{\lambda(t-a)} K(a, \lambda)+\bar{S} e^{\lambda(t-\bar{\tau})} K(\bar{\tau}, \lambda)\left(\frac{g^{+}}{g^{-}}-1\right) H(a-\bar{\tau}) .
$$

Next, we compute $D_{2} \mathcal{F}(a, \bar{S}) \psi$ from the representation

$$
\mathcal{F}(a, \psi)=e^{-\int_{0}^{\tilde{\tau}(a, \psi)} \mu(x(\sigma ; a, \psi), \psi(-a+\sigma)) d \sigma-\int_{\tilde{\tau}(a, \psi)}^{a} \mu(x(\sigma ; a, \psi), \psi(-a+\sigma)) d \sigma} .
$$

If we formally linearize (9) and use (A.9), we deduce that

$$
\begin{aligned}
D_{2} \mathcal{F}(a, \bar{S}) \psi & =\left(\mu^{+}-\mu^{-}\right) \mathcal{F}(a) D_{2} \tilde{\tau}(a, \bar{S}) \psi+\zeta(a ; a, \psi) \\
& =-\frac{\mu^{+}-\mu^{-}}{g^{-}} \mathcal{F}(a) \eta(\bar{\tau} ; a, \psi)+\zeta(a ; a, \psi),
\end{aligned}
$$

where $\zeta(\tau)=\zeta(\tau ; a, \psi)$ satisfies

$$
\zeta^{\prime}(\tau)=-\mu(\tau) \zeta(\tau)-\mu_{1}(\tau) \eta(\tau) \mathcal{F}(\tau)-\mu_{2}(\tau) \psi(-a+\tau) \mathcal{F}(\tau)
$$

and $\eta$ satisfies (A.3). Again, for later use, we plug in $\psi:=z_{t}, z(\alpha)=\bar{S} e^{\lambda \alpha}, \eta(\tau)=$ $\bar{S} e^{\lambda(t-a)} K(\tau, \lambda)$ and redefine for this case $\zeta(\tau):=\bar{S} e^{\lambda(t-a)} L(\tau, \lambda)$, where $L$ is defined via (38) for $a_{0}:=0$ and $l^{0}:=0$. One then has that

$$
D_{2} \mathcal{F}(a, \bar{S}) z_{t}=-\frac{\mu^{+}-\mu^{-}}{g^{-}} \mathcal{F}(a) \bar{S} e^{\lambda(t-a)} K(\bar{\tau}, \lambda)+\bar{S} e^{\lambda(t-a)} L(a, \lambda) .
$$


It remains to compute $D a_{A}(\bar{S}) \psi$. If we differentiate (5), we get

$$
g^{-} D a_{A}(\bar{S}) \psi+D_{2} X(\bar{\tau}, \bar{S}) \psi=0 .
$$

Hence for the exponential trial solutions we get

$$
D a_{A}(\bar{S}) z_{t}=-\frac{\eta\left(\bar{\tau} ; \bar{\tau}, z_{t}\right)}{g^{-}}=-\frac{1}{g^{-}} \bar{S} e^{\lambda(t-\bar{\tau})} K(\bar{\tau}, \lambda) .
$$

Now we are ready to plug $y(t)=\bar{b} e^{\lambda t}, z(t)=\bar{S} e^{\lambda t}$ into (A.1)-(A.2), use (A.11), (A.13), and (A.14) and divide the resulting equations by $e^{\lambda t}$ to get

$$
\begin{aligned}
\bar{b}= & \bar{b} \int_{\bar{\tau}}^{h} \beta(a) \mathcal{F}(a) e^{-\lambda a} d a+\frac{\overline{b S} \beta^{+}}{g^{-}} e^{-\lambda \bar{\tau}} K(\bar{\tau}, \lambda) \mathcal{F}(\bar{\tau}) \\
& +\overline{b S} \int_{\bar{\tau}}^{h} \beta_{2}(a) \mathcal{F}(a) d a+\overline{b S} \int_{\bar{\tau}}^{h} \mathcal{F}(a) \beta_{1}(a) e^{-\lambda a} K(a, \lambda) d a \\
& +\overline{b S} e^{-\lambda \bar{\tau}} K(\bar{\tau}, \lambda)\left(\frac{g^{+}}{g^{-}}-1\right) \int_{\bar{\tau}}^{h} \beta_{1}(a) \mathcal{F}(a) d a \\
& +\overline{b S} \frac{\mu^{-}-\mu^{+}}{g^{-}} K(\bar{\tau}, \lambda) \int_{\bar{\tau}}^{h} e^{-\lambda a} \beta(a) \mathcal{F}(a) d a \\
& +\overline{b S} \int_{\bar{\tau}}^{h} \beta(a) e^{-\lambda a} L(a, \lambda) d a, \\
\lambda \bar{S}= & f^{\prime}(\bar{S}) \bar{S}-\bar{b} \int_{0}^{h} \gamma(a) \mathcal{F}(a) e^{-\lambda a} d a-\frac{\overline{b S}\left(\gamma^{+}-\gamma^{-}\right)}{g^{-}} e^{-\lambda \bar{\tau}} K(\bar{\tau}, \lambda) \mathcal{F}(\bar{\tau}) \\
& -\overline{b S} \int_{0}^{h} \gamma_{2}(a) \mathcal{F}(a) d a-\overline{b S} \int_{0}^{h} \mathcal{F}(a) \gamma_{1}(a) e^{-\lambda a} K(a, \lambda) d a \\
& -\overline{b S} e^{-\lambda \bar{\tau}} K(\bar{\tau}, \lambda)\left(\frac{g^{+}}{g^{-}}-1\right) \int_{\bar{\tau}}^{h} \gamma_{1}(a) \mathcal{F}(a) d a \\
& -\overline{b S} \frac{\mu^{-}-\mu^{+}}{g^{-}} K(\bar{\tau}, \lambda) \int_{\bar{\tau}}^{h} e^{-\lambda a} \gamma(a) \mathcal{F}(a) d a \\
& -\overline{b S} \int_{0}^{h} \gamma(a) e^{-\lambda a} L(a, \lambda) d a . \\
& \\
& \\
&
\end{aligned}
$$

If one uses the definition of the $\Psi^{i}$ as given in (34)-(36), one sees that Eqs. (A.15)-(A.16) are equivalent to the condition

$$
0=\left(M(\bar{b}, \bar{S}, \lambda)-\left(\begin{array}{ll}
1 & 0 \\
0 & \lambda
\end{array}\right)\right)\left(\frac{\bar{b}}{\bar{S}}\right)
$$

with $M(\bar{b}, \bar{S}, \lambda)$ as defined in (27). This concludes the derivation of the characteristic equation. 


\section{References}

Allgower, E.L., Georg, K., 1990. Numerical Continuation Methods, an Introduction. SCM, vol. 13. Springer, Berlin.

de Roos, A.M., 1997. A gentle introduction to models of physiologically structured populations. In: Tuljapurkar, S., Caswell, H. (Eds.), Structured-Population Models in Marine, Terrestrial, and Freshwater Systems, pp. 119-204. Chapman and Hall, New York.

de Roos, A.M., Metz, J.A.J., Evers, E., Leipoldt, A., 1990. A size-dependent predator prey interaction: Who pursues whom? J. Math. Biol. 28, 609-643.

de Roos, A.M., Diekmann, O., Gyllenberg, M., Metz, J.A.J., Nakaoka, S., 2009. Daphnia revisited. Submitted to J. Math. Biol.

Diekmann, O., van Gils, S., Verduyn Lunel, S.M., Walther, H.-O., 1995. Delay Equations, Functional-, Complex-, and Nonlinear Analysis. Springer, New York.

Diekmann, O., Getto, P., Gyllenberg, M., 2007. Stability and bifurcation analysis of Volterra functional equations in the light of suns and star. SIAM J. Math. Anal. 39(4), 1023-1069.

Hale, J., 1977. Functional Differential Equations. Springer, New York.

Hairer, E., Nørsett, S.P., Wanner, G., 1987. Solving Ordinary Differential Equations I. Nonstiff Problems. Springer Series in Computational Mathematics, vol. 8. Springer, Berlin.

Kirkilionis, M.A., Diekmann, O., Lisser, B., Nool, M., Sommeijer, B., de Roos, A.M., 2001. Numerical continuation of equilibria of physiologically structured population models. I. Theory. Math. Mod. Meth. Appl. Sci. 11(6), 1101-1127.

Kuznetsov, Y.A., 1994. Elements of Applied Bifurcation Theory. Springer, New York.

Metz, J.A.J., Diekmann, O. (Eds.), 1986. The Dynamics of Physiologically Structured Populations. Lecture Notes in Biomathematics, vol. 68. Springer, Berlin.

Rosenzweig, M.L., 1971. Paradox of enrichment: destabilization of exploitation ecosystems in ecological time. Science (Wash., DC) 171, 385-387. 